\title{
ÉCONOMIE ET POLITIQUE Les marchés du crédit à Paris, 1750-1840
}

Philip Hoffman, Gilles Postel-Vinay, Jean-Laurent Rosenthal

En rejetant l'événement et le temps court pour le temps long, la tradition qu'ont longtemps illustrée les Annales tendait à réduire la politique à l'économie et à oublier l'histoire des États au profit d'une histoire des sociétés. Il se trouve que cette approche n'a inspiré que peu d'études consacrées à l'évolution pluriséculaire des rapports de crédit. Pourtant une telle histoire aurait certainement un sens. Elle permettrait de distinguer deux grands circuits : celui des marchands, bien sûr, qui traitait avant tout de crédit à court terme, mais aussi un autre circuit qui, lui, était spécialisé dans des affaires plus longues. Ce dernier est moins connu bien qu'il ait été omniprésent puisqu'il liait tous ceux qui, à un moment de leur cycle de vie, épargnaient et plaçaient ou, au contraire, empruntaient pour une certaine durée au moyen d'engagements contractuels. A la période moderne, ce circuit était extrêmement diffus et, si l'on en juge par des exemples français du XVIII ${ }^{\mathrm{e}}$ ou du XIX ${ }^{\mathrm{e}}$ siècle, il atteignait au moins une forte minorité de la population sinon la majorité. Dans le cas français - qui était loin d'être unique en Europe - il s'était organisé autour des réseaux notariaux et c'était surtout par leur intermédiaire que circulait la fraction de l'épargne qui ne restait pas à l'intérieur des familles. Il faudrait alors analyser, outre la coexistence de ces deux circuits, les conditions et les règles de fonctionnement du second ainsi que ses performances pour comprendre son long maintien et, par là même, l'émergence tardive d'organisation de substitution comme les banques.

Mais cette démarche ne serait qu'en partie satisfaisante. Car au moins autant qu'aux permanences organisationnelles locales et aux lentes évolutions des déterminants de l'épargne, l'histoire des circuits de crédit renvoie à une autre dimension. Elle était intimement liée à des conjonctures plus vastes mais aussi plus instables et d'abord à la situation politique, ne serait-ce qu'en raison des à-coups que l'État lui faisait supporter au gré des besoins des finances publiques. Elle dépendait également des conditions réglementaires changeantes et, plus encore, des bouleversements introduits 


\section{MODERNISATION DES SOCIÉTÉS TRADITIONNELLES}

par certains événements politiques majeurs comme la Révolution française. Pour examiner ces questions, nous nous attacherons à l'histoire du marché parisien du crédit entre 1750 et 1840 . Le réseau formé par les notaires de la capitale était le plus important du pays. Mais il était aussi particulièrement exposé puisque sa position dominante s'explique à la fois par la richesse exceptionnelle de sa clientèle et parce que les études jouaient un rôle d'intermédiaire privilégié - jusqu'à la fin de l'Ancien Régime tout au moins - dans le placement des emprunts publics.

Après avoir présenté comment ont été établies les informations nécessaires, nous analyserons dans quelle mesure l'évolution de ce marché sur un siècle a été marquée par ses liens avec le crédit public et quel impact eut la Révolution sur les formes et l'intensité des échanges financiers.

\section{Sur la méthode}

Paradoxalement, l'analyse du fonctionnement du crédit ancien peut s'appuyer sur une documentation d'une abondance et d'une précision dont les sources ultérieures sont loin de fournir l'équivalent puisque les circuits notariaux étaient tenus de conserver les contrats de prêts qui passaient par eux. En revanche et quelle que soit sa qualité, l'information ainsi rassemblée présente au moins deux inconvénients. Ses lacunes d'abord. Prêteurs et emprunteurs se dispensaient de recourir aux notaires pour une partie de leurs transactions (pour certains crédits intra-familiaux par exemple). De plus, les études n'avaient pas à garder la totalité des contrats qu'elles passaient (contrats en brevet). Les prêts qui ne laissaient pas de trace dans les minutiers, étaient de durée assez courte et avaient, en général, d'autres fonctions que ceux qu'ont conservés les archives notariales ${ }^{1}$ mais ils pouvaient aussi se substituer à eux selon des modalités que l'on ne peut observer directement. D'autre part, la masse de la documentation exclut l'exhaustivité. Une analyse des prêts réalisés chez les notaires parisiens pendant un siècle ne se conçoit donc qu'à condition de procéder par sondage.

Nous avons ainsi été amenés à sélectionner un petit nombre d'études. En nous appuyant sur le dépouillement des actes notariés effectué par les Archives Nationales pour l'année 1751, nous en avons retenu neuf qui paraissaient représentatives au début de la période considérée afin de suivre l'ensemble de leurs prêts sur un siècle ${ }^{2}$. Pour disposer des renseignements nécessaires - le nombre des différents types d'affaires, leur montant et leur durée - l'une des sources produites par le notariat fournissait un moyen commode. En sus des minutes qu'elles devaient conserver, les études étaient en effet tenues de consigner un bref descriptif des actes qu'elles passaient dans des répertoires systématiques. Par leur sécheresse même, ces registres

1. Il s'agissait de crédit commercial, de crédit de campagne ou de crédit à la consommation. Les sondages provinciaux dans le contrôle des actes amènent à penser que les transactions réalisées sous la forme de billets et de lettres de change, bien que très nombreuses, n'ont pu contribuer à plus du tiers du volume des prêts et à beaucoup moins de l'encours.

2. Études IX, XXI, XXVII, XLIII, LXII, LXX, LXXVIII, CXV et CXVII. Ces sources comme les suivantes sont conservées aux Archives Nationales (AN). 
permettent de rassembler des informations continues sur l'évolution longue du crédit notarié dans la capitale. Plus précisément, nous suivons bien ainsi sur toute la période le nombre des actes mais nous ignorons leur montant et leur durée jusqu'à ce que les répertoires en fassent état à l'extrême fin du $\mathrm{XVIII}^{\mathrm{e}}$ siècle. Pour combler cette lacune, il fallait revenir aux minutes ellesmêmes. Nous avons admis que le montant et la durée des différents prêts pouvaient être approchés par les renseignements correspondants issus du dépouillement des actes passés par sept des neuf études pendant les années $1740,1760,1780$ et $1788^{3}$.

Si l'on cerne ainsi l'évolution longue d'un échantillon représentatif pour l'année 1751, rien ne garantit que sa contribution au crédit parisien à moyen et long terme soit demeurée stable au cours du temps. Nous avons donc confronté ses résultats à ceux de 21 études supplémentaires pour les années 1780,1820 et $1840^{4}$. Mise à part cette dernière année pour laquelle nos neuf études ont une activité inférieure à la moyenne, les prêts qu'elles réalisent représentent une proportion presque constante du crédit parisien. Sur cette base, il a paru légitime d'extrapoler leurs résultats à l'ensemble de la capitale en les redressant pour la fin de la période considérée.

Il est certain qu'en raison de la structure décentralisée du crédit notarial, pareille information devrait faire l'objet d'une analyse détaillée étude par étude. Dans le cadre que les notaires offraient à leur clientèle, les individus désirant emprunter et les bailleurs de fonds potentiels ne se rencontraient pas sans difficulté. Si le montant d'un prêt, sa date et ses conditions devaient convenir à l'emprunteur, ils dépendaient aussi des moyens que l'étude était à même de mobiliser au moment voulu. Or les notaires de la capitale ne brassaient pas une masse de prêts telle que les demandes tantôt fortes tantôt faibles de leurs clients aient été en quelque sorte lissées par la loi des grands nombres. Très concentré, le crédit à Paris portait sur relativement peu de contrats. Une étude réalisait en moyenne cinq prêts par mois pendant la seconde moitié du XVIII ${ }^{e}$ siècle et son activité financière, souvent irrégulière, pouvait être soumise à de brusques à-coups qui lui posaient de délicats problèmes de fonctionnement. Quoique cet aspect ait eu des conséquences importantes, nous en ferons abstraction ici pour mettre d'abord l'accent sur les caractéristiques de l'évolution à long terme du système de crédit notarial. Dans cette optique, nous nous en tiendrons donc à l'examen des résultats agrégés de nos études prises comme un tout.

L'activité de crédit de cet échantillon - et, à travers lui, celle du marché parisien - doit d'autre part être replacée dans son contexte géographique et économique. En ce sens, il importe tout spécialement de situer le poids relatif de la capitale dans le crédit notarial de l'ensemble du pays (voire au delà),

3. Jusqu'à la fin du XviII ${ }^{\circ}$ siècle, les répertoires ne contiennent que le nom des parties et le type de l'acte. Afin de compléter l'information, nous avons calculé la durée et le montant moyen de chaque type de prêt au cours du temps à partir d'un sondage portant sur les prêts réalisés dans les études IX, XXI, XLIII, LXII, LXX, LXXVIII et CXV. Voir le tableau A de l'Annexe.

4. Études I à VIII, X à XX, XCIII, CXI. Les résultats des neuf études de l'échantillon retenu peuvent ainsi être pondérés pour estimer le volume global de l'activité du crédit notarié dans la capitale. Les coefficients sont donnés dans le tableau B de l'Annexe. 


\section{MODERNISATION DES SOCIÉTÉS TRADITIONNELLES}

afin de mieux apprécier la portée des relations qui pouvaient se nouer de place à place. Ces questions étant pour le moins mal connues, un tel programme paraîtra à première vue incertain, voire illusoire. Quitte à n'en attendre que des ordres de grandeur, plaidons pourtant qu'une tentative en ce sens ne semble pas tout à fait impossible, à deux conditions.

La première est de se donner un indicateur qui permette de comparer de manière satisfaisante l'activité de crédit en différents lieux tout en tenant compte de la diversité des formes de prêts. En effet, sous l'Ancien Régime, les circuits notariaux utilisaient trois grands types de contrats. Les rentes perpétuelles d'abord, qui étaient des placements de père de famille. Elles offraient au prêteur les conditions légales des immeubles et un taux d'intérêt prescrit. En revanche le bailleur de fonds qui y avait recours perdait tout contrôle sur la durée de son placement puisque le remboursement se faisait à la volonté de l'emprunteur. Les rentes viagères ensuite. Elles jouissaient des mêmes conditions légales mais limitaient l'engagement à la durée de la vie d'un individu, ce qui revenait implicitement à un remboursement progressif du capital. Les obligations enfin. Celles-ci étaient plus brèves et fixaient la durée du prêt ; en contrepartie elles ne permettaient pas de stipuler en clair le taux d'intérêt pratiqué. Comme la durée et le montant des transactions variaient selon les lieux, les périodes et les types de contrat, il ne saurait suffire de raisonner en confrontant de région à région le nombre de prêts réalisés ou le volume des sommes avancées. Le mieux serait de s'appuyer sur la valeur des encours si l'objectif n'était pas hors d'atteinte (pour la reconstituer il faudrait connaître la durée effective des prêts sur des périodes très longues). A défaut, nous utiliserons donc une notion intermédiaire qui tient compte à la fois du montant et de la durée des engagements. Nous nous attacherons ainsi moins au nombre des actes ou au volume des prêts qu'aux «stocks », en entendant par là la somme des prêts pondérés par leur durée. Il va de soi qu'il ne revenait pas au même d'engager, disons, un million de livres en rentes perpétuelles ou en obligations sur deux, trois ou quatre ans. L'indicateur - qui équivaut en fait à l'encours dans le cas particulier où le montant et la durée des prêts seraient stables au cours du temps - a notamment l'avantage de rendre comparable l'activité de différents marchés sur la base de coupes annuelles. Nous présenterons ici deux coupes de ce type qui prennent la mesure des différents marchés, l'une à la fin de l'Ancien Régime, en 1780, l'autre en 1840.

La seconde condition à respecter découle de la très inégale répartition du crédit dans l'espace. A la fin de l'Ancien Régime, faute de disposer de quelque repère que ce soit, il paraît au moins nécessaire de scinder le pays en trois sous-ensembles. La capitale tout d'abord qui, par sa richesse et son rôle dans les émissions publiques, tenait une place à part. Les grandes villes ensuite (par commodité elles seront ici arbitrairement définies comme les villes de plus de 15000 habitants). Les campagnes, enfin, avec leur maillage hiérarchisé de bourgs et de petits centres urbains. Si l'on admet cette tripartition, on peut alors procéder par approximations successives.

Pour la capitale, nous nous appuierons sur le sondage portant sur trente études, soit le quart du notariat parisien. Sans doute, bien que leur clientèle ait été assez fidèle, ces sortes de bureaux de crédit qu'étaient alors les études 
n'avaient pas une activité stable au cours du temps et, sur longue période, tels notaires périclitaient alors que d'autres connaissaient une ascension remarquable. Mais l'échantillon paraît suffisant pour extrapoler ses résultats aux 122 études que compte la capitale et donner une évaluation fiable du stock de prêts à Paris aux dates considérées.

Les autres grandes villes offraient certainement un éventail de situations assez ouvert. C'est pourquoi, excluant à la fois les plus actives et les moins prospères, nous avons supposé qu'une ville comme Dijon pouvait représenter un cas moyen. Le stock de prêts par habitant dans la capitale bourguignonne a donc été étendu à toute les villes de plus de 15000 habitants, Paris exclu.

La contribution des autres marchés est plus délicate à évaluer tant les disparités étaient fortes d'un lieu à l'autre. Pour cette première tentative, nous avons choisi six marchés locaux. Tous présentent la caractéristique courante à l'époque de s'étendre sur une aire assez restreinte rassemblant de 10 à 20000 personnes, soit un ensemble de villages groupés autour d'un petit centre, en l'occurrence Bar-sur-Aube, Château-Thierry, l'Isle-sur-la-Sorgue, Maubeuge, Nuits-Saint-Georges et Privas. D'autre part, dispersés du nord au sud du pays, chacun avec ses spécialisations particulières, ils reflètent des situations très différenciées, et pas seulement par les niveaux de revenu. Le crédit variait trop d'une zone à l'autre pour dépendre uniquement de l'inégale vitalité des économies locales. A la fin de l'Ancien Régime par exemple, le montant par tête des engagements financiers sextuplait quand on passait du Vivarais (Privas) au Comtat Venaissin (l'Isle-sur-la-Sorgue). Ce sont là deux cas extrêmes, mais, puisque les revenus moyens n'étaient sûrement pas aussi éloignés, il faut supposer qu'un tel écart découlait pour partie de facteurs d'un autre ordre et notamment des structures foncières, des rapports de parenté et des conditions juridiques ${ }^{5}$. En un sens, une si grande hétérogénéité rend nécessairement fragile une estimation globale de l'activité des marchés locaux. Mais comme, ramené au nombre d'habitants, le crédit était là toujours beaucoup moins abondant que dans les grands centres, il ne paraît pas déraisonnable d'étendre à l'ensemble de la population des campagnes et des petites villes l'engagement moyen par tête sur ces six marchés ${ }^{6}$.

Pour imparfaite qu'elle soit, cette esquisse d'esquisse fait apparaître de fortes permanences et des hiérarchies trop nettes pour être mises en doute. En 1780, comme un demi-siècle plus tard, ces circuits de crédit décentralisés distribuent des sommes très considérables. Le montant estimé des prêts réalisés sur une année ne correspond certes pas à la formation d'une épargne

5. J.-C. Perror, Genèse d'une ville moderne. Caen au xvilr siècle, Paris-La Haye, Mouton, 1975, p. 464.

6. Ce que l'on sait d'autres marchés locaux conforte l'estimation proposée. Pour 1840, celle-ci paraît d'ailleurs préférable à l'évaluation avancée à l'époque par Martin du Nord (selon lequel, " la somme annuelle des prêts hypothécaires " se serait alors élevée à quelque 500 millions, cf. Documents relatifs au régime hypothécaire..., Paris, 1844, p. V). En outre, bien que l'activité locale ait souvent beaucoup varié d'une année sur l'autre - et plus spécialement à la campagne - la prise en compte d'une période d'observation plus longue ne modifie pas significativement les résultats obtenus. 


\section{MODERNISATION DES SOCIÉTÉS TRADITIONNELLES}

nette (ne serait-ce que parce qu'une partie des prêts remboursés étaient replacés) mais il est malgré tout frappant qu'il approche à ces deux dates le dixième de la valeur du PIB. D'autre part, la répartition du stock des prêts entre urbains et ruraux est demeurée relativement stable. Si les grandes villes occupent une place privilégiée eu égard à leur population, le reste du pays, et donc d'abord les campagnes, n'en détient pas moins pendant toute la période un peu plus de la moitié du stock des prêts (voir tableau 1).

TABLEAU 1 - Répartition spatiale des volumes et des stocks de prêts notariés réalisés entre particuliers en 1780 et 1840

\begin{tabular}{lrrrrrr} 
& $\begin{array}{c}\text { Prêts moyens } \\
\text { (livres puis francs) }\end{array}$ & $\begin{array}{c}\text { Volumes estimés } \\
\text { (millions) }\end{array}$ & \multicolumn{2}{c}{$\begin{array}{c}\text { Stocks estimés } \\
\text { (milliards) }\end{array}$} \\
& \multicolumn{2}{c}{-} & \multicolumn{2}{c}{-} & \multicolumn{2}{c}{-} \\
& 1780 & 1840 & 1780 & 1840 & 1780 & 1840 \\
1 a Paris & 14619 & 29270 & 135 & 89 & 1,28 & 0,47 \\
1 b Autres villes & 1674 & 3515 & 56 & 270 & 0,79 & 1,55 \\
1 Total grandes villes & 4447 & 4496 & 191 & 359 & 2,07 & 2,02 \\
2 Autres = "ruraux" & 513 & 1478 & 215 & 597 & 2,10 & 2,39 \\
3 Ensemble & 905 & 1962 & 406 & 956 & 4,17 & 4,41 \\
2/3 & $57 \%$ & $75 \%$ & $53 \%$ & $62 \%$ & $50 \%$ & $54 \%$
\end{tabular}

Sources : voir texte.

De même que le rapport entre le stock des prêts urbains et ruraux n'a guère changé pendant ce demi-siècle, la taille des actes est, elle aussi, toujours restée bien plus considérable dans les grandes villes. Enfin le marché parisien garde un certain nombre de traits spécifiques. Il se singularise en particulier par le montant exceptionnel des prêts qui y étaient réalisés ainsi que par une quasi-absence de saisonnalité. Il connaissait naturellement de multiples variations dans le court terme mais elles résultaient des demandes aléatoires d'un petit groupe de très gros clients bien plus qu'elles ne répondaient au rythme des saisons. Au XVIII ${ }^{\mathrm{e}}$ siècle, l'activité du premier semestre était, en moyenne, un peu plus soutenue que celle du second pour certains types de prêts comme les obligations et les rentes conclues entre particuliers. Mais les fluctuations restaient faibles et irrégulières au point d'être fréquemment atypiques. Les emprunts publics et les viagères privées suivaient, quant à eux, des mouvements erratiques au gré des besoins des emprunteurs. Au $\mathrm{XIX}^{\mathrm{c}}$ siècle, a fortiori, toute trace de saisonnalité se perd ${ }^{7}$. L'activité finan-

7. S'il ne reposait que sur les contrats passés chez neuf notaires, ce constat pourrait n'être qu'un biais d'échantillon. Quelques études seraient susceptibles d'ignorer des fluctuations courtes qui seraient éventuellement plus marquées dans d'autres (voir en particulier, J.-P. PorsSON, Notaires et société, Paris, Economica, 1985). Il se pourrait aussi que l'échantillon souligne indûment les à-coups introduits par les demandes de quelques très gros clients qui s'estomperaient si l'on disposait d'une base d'observation moins étroite. Mais les coupes plus étendues effectuées pour les années 1751,1780 et 1820 ne révèlent pas non plus de saisonnalité. La situation était différente sur les marchés locaux (cf. J. L. LAFFONT éd., Problèmes et méthode d'ana- 
cière parisienne s'était d'ores et déjà largement affranchie des contraintes liées à la respiration du monde agricole.

Mais ces permanences ne doivent pas masquer de véritables bouleversements que reflètent aussi, fût-ce grossièrement, les indications du tableau 1. Elles soulignent combien la part de Paris faiblit dans la première moitié du $\mathrm{XIX}^{\mathrm{e}}$ siècle, inversant ainsi une tendance longue à la centralisation. Que l'on considère le volume des prêts et, plus encore, les stocks, l'activité de crédit dans la capitale chute brutalement en un demi-siècle. La rupture est si nette qu'elle impose de déplacer l'analyse vers l'étude des changements intervenus et vers les liens entre le fonctionnement du crédit et la conjoncture institutionnelle ou politique dont le rôle apparaît essentiel, tout spécialement lors d'un événement majeur comme la Révolution (voir figure 1$)^{8}$.

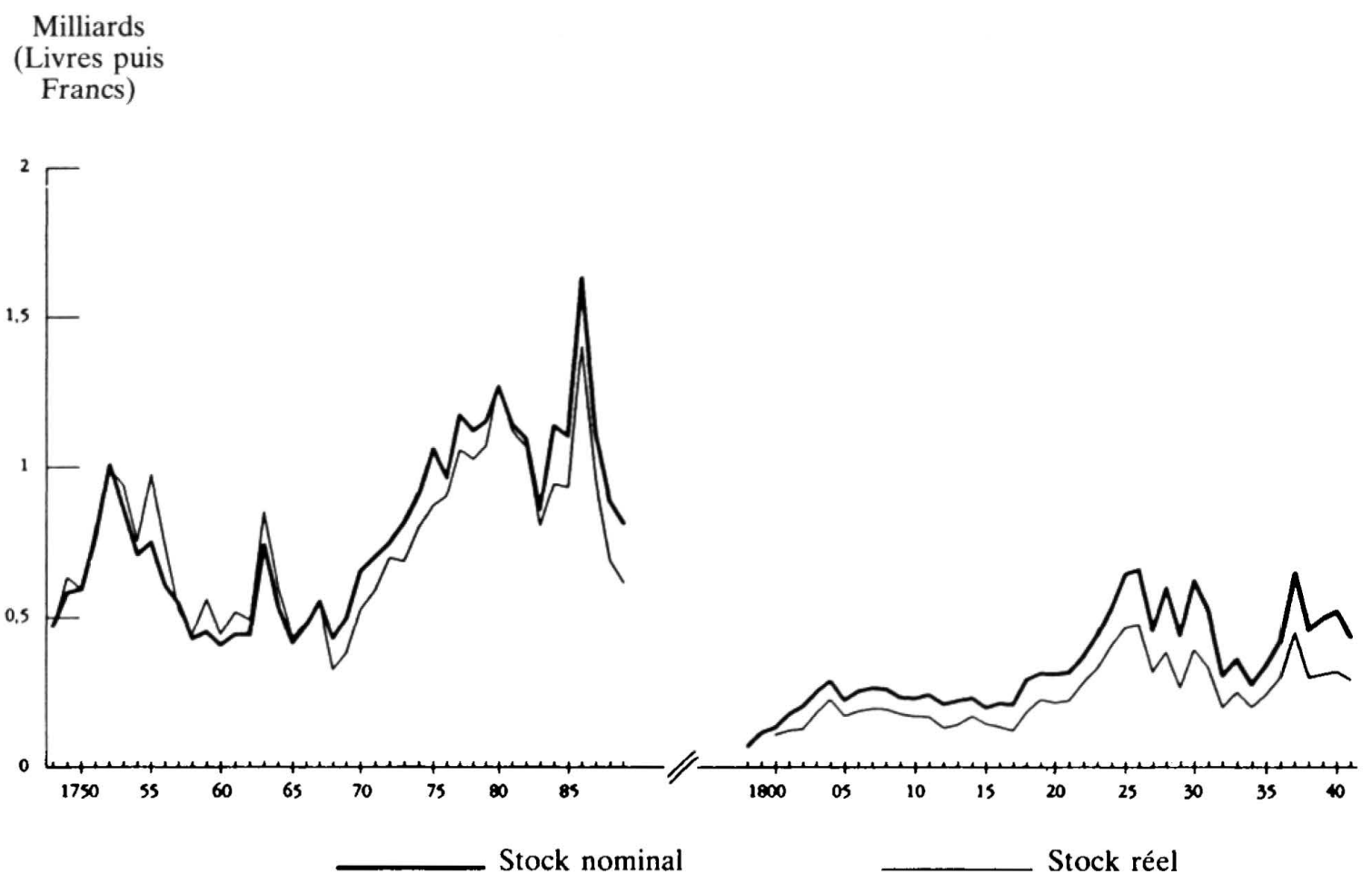

FIG. 1. - Stocks estimés des prêts privés à Paris, valeurs annuelles, nominales et déflatées

\section{L'évolution du marché au XVIIP siècle}

Le volume du crédit qui transitait par le notariat parisien est allé croissant jusqu'à la fin de l'Ancien Régime. Cette tendance favorable n'excluait pas, loin de là, de fortes variations de moyen terme dont les liens aux flux et

lyse historique de l'activité notariale ( $X V^{e}-X I X^{e}$ siècles), Toulouse, Presses Universitaires du Mirail, 1991.

8. Les résultats sont établis à partir des neuf études suivies dont l'activité a été extrapolée à l'ensemble de la capitale après avoir pondéré l'échantillon en utilisant les résultats des quatre coupes présentées dans le tableau B de l'Annexe. 
aux reflux de l'activité économique ne peuvent malheureusement pas être analysés faute d'information. Il n'en reste pas moins que la croissance globale pendant les quatre dernières décennies du siècle a été remarquable. Dira-t-on que les prêts entre particuliers se sont alors accrus de $2 \%$ chaque année ? Il est plus réaliste de souligner que, déflatée ${ }^{9}$, la hausse a été moitié moindre et qu'elle semble souvent fragile et mal assurée. Tel fut le cas pendant les années 1780 où les affaires ont marqué le pas. Pourtant, la performance d'ensemble demeure plus qu'honorable et, à travers hauts et bas, le secteur paraît bien engagé sur une trajectoire ascendante (et plus rapidement ascendante que le reste de l'économie).

Le constat a d'autant plus d'importance que la place de Paris avait un poids considérable à l'échelle du pays. Affaires publiques exclues, le quart de l'activité financière se serait alors négocié dans les études parisiennes, soit plus que dans toutes les autres grandes villes. A l'opposé, l'ensemble des petits centres urbains et des ruraux n'auraient retenu qu'un peu plus de la moitié du crédit privé (cf. supra tableau 1). Présent de manière diffuse dans les campagnes, celui-ci se rassemblait ainsi dans le monde urbain et d'abord à Paris. Comme l'activité économique de la capitale était loin de représenter une telle proportion du produit national, il faut admettre que la concentration du crédit était un phénomène ancien et bien antérieur au développement des banques. Si l'on compare marché par marché le stock de crédit par tête, il était en 1780 de 2148 livres dans la capitale, de 462 dans une ville comme Dijon et de 82 livres en moyenne pour les habitants des six zones rurales examinées (voir figure 2).

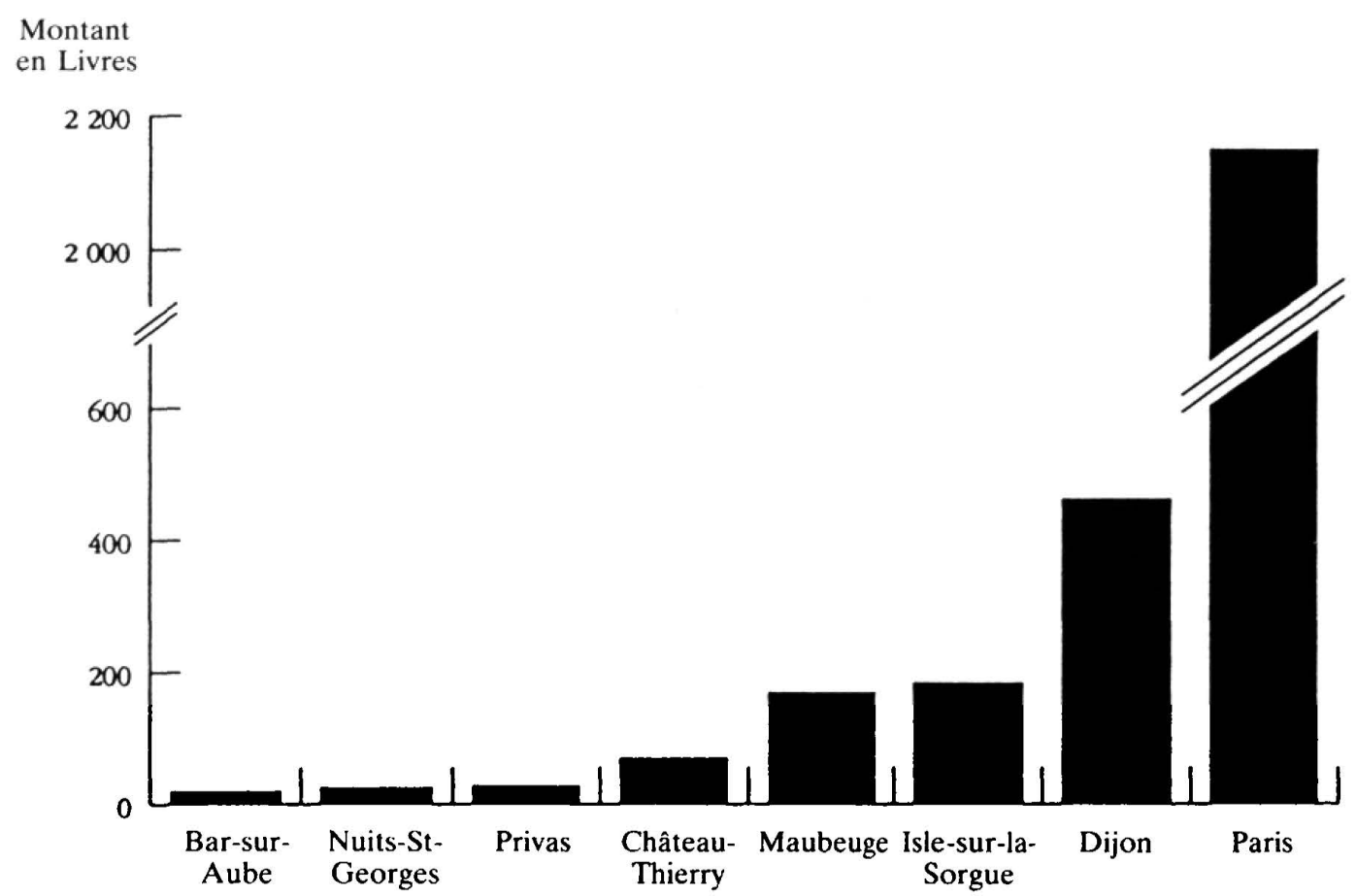

Fig. 2. - Stocks des prêts privés par tête, sur huit marchés, en 1780

9. Nous remercions D. Weir de nous avoir communiqué l'indice des prix à la consommation qu'il a établi et qui présentait pour nous l'avantage de compléter et de prolonger les séries labroussiennes. 
En un sens, la position tout à fait prédominante de la capitale reflétait un certain isolement d'un marché assez puissant et riche pour se satisfaire de ses propres ressources. Et, de fait, la grande majorité des transactions se faisaient alors entre Parisiens. Pas exclusivement toutefois. Prêteurs et emprunteurs provinciaux et étrangers étaient présents sur le marché de la capitale, pour les affaires privées et plus encore pour participer aux emprunts publics qui bien que très discontinus représentaient bon an mal an environ le tiers des placements qui y étaient effectués (voir tableau 2).

TABleAu 2 - Origine des prêteurs et des emprunteurs chez les notaires parisiens

\begin{tabular}{|c|c|c|c|c|c|c|c|c|c|}
\hline & & & 175 & -1780 & & & 179 & 1840 & \\
\hline Ori & gines & $\mathrm{Nb}$ de & itrats & Sommes & gagées & $\mathrm{Nb}$ de & itrats & Sommes & gagées \\
\hline Prêteurs & Débit. & Nombre & $\%$ & Valeur & $\%$ & Nombre & $\%$ & Valeur & $\%$ \\
\hline - & - & - & - & - & - & - & - & - & - \\
\hline Paris & Paris & 1329 & 72 & 17761 & 76 & 334 & 53 & 5490 & 55 \\
\hline Paris & Ailleurs & 194 & 10 & 1682 & 7 & 153 & 24 & 2255 & 23 \\
\hline Ailleurs & Paris & 224 & 12 & 3137 & 13 & 97 & 15 & 1654 & 17 \\
\hline Ailleurs & Ailleurs & 111 & 6 & 886 & 4 & 43 & 7 & 508 & 5 \\
\hline Paris & État & 1053 & 64 & 4878 & 72 & & & & \\
\hline Ailleurs & État & 593 & 36 & 1890 & 28 & & & & \\
\hline
\end{tabular}

Valeurs en milliers de Livres/Francs. "Ailleurs" désigne toute résidence autre que Paris (la région parisienne, le reste de la France et l'étranger). Les résidences inconnues ont été exclues. Sondage sur sept études ${ }^{10}$.

Comme de nombreux réseaux locaux, le marché parisien pouvait ainsi en cas de besoin être soutenu par d'autres, proches ou moins proches, auxquels il se trouvait lié par divers groupes d'intermédiaires. Or la capacité d'un marché à surmonter des crises passagères dépendait de son aptitude à attirer des fonds de l'extérieur - un problème critique pour Paris où les émissions publiques étaient toujours susceptibles de provoquer de forts déséquilibres. Mais, précisément, la capitale était un marché fort, mieux que d'autres à même d'attirer des ressources extérieures. Un bailleur de fonds en quête de placements avait sûrement plus de mal à y recueillir des renseignements fiables que sur un marché étroit où les emprunteurs potentiels étaient parfaitement identifiés ${ }^{11}$, mais Paris n'en offrait pas moins une palette incomparable de demandeurs de crédit présentant les meilleures garanties. Les plus aisés y disposaient normalement de revenus d'origines assez diversifiées pour être à l'abri des aléas et pouvoir assurer le service régulier de leurs dettes. Quand la demande de l'État se faisait plus intense, un tel marché pouvait donc aspirer vers lui des fonds venus de province ou d'ailleurs. La capitale tirait aussi avantage de sa position particulière dans les trois grands flux financiers de la France d'Ancien Régime. Paris se trouvait au centre du

10. Ph. Hoffman, G. Postel-Vinay, J.-L. Rosenthal, "Private Credit Markets in Paris, 1690-1840 », The Journal of Economic History, Vol. 52, n² (June 1992), pp. 293-306.

11. Ph. Hoffman, G. Postel-Vinay, J.-L. Rosenthal, ibid. 


\section{MODERNISATION DES SOCIÉTÉS TRADITIONNELLES}

circuit lié aux finances publiques et de bien des circuits marchands. D'autre part, les plus riches de ses habitants étaient aussi possessionnés ailleurs. A la tête d'actifs et de passifs géographiquement diversifiés, ils avaient donc couramment à transférer des fonds entre Paris et la province sans changer la taille totale de leur portefeuille. Le dernier de ces circuits portait autant sur des engagements brefs que sur des investissements durables. Les deux premiers concernaient plutôt des affaires de court terme mais marchands et bourgeois parisiens, outre leurs affaires commerciales, participaient aussi activement, et d'abord comme prêteurs, au crédit notarial qui était surtout à moyen et long terme. Tous ces circuits entremêlés offraient donc la possibilité de multiples glissements entre les différents compartiments du système financier. Il y avait là une source de souplesse qui pourrait avoir permis au marché financier parisien de poursuivre sa croissance tout en répondant largement à des émissions publiques aussi irrégulières que massives. Leurs contrecoups auraient été atténués par la mobilisation de sommes thésaurisées localement ou par des apports extérieurs mais aussi par le report vers des placements longs de ressources engagées dans les circuits du court terme.

A ces mouvements, il faut d'ailleurs ajouter la possibilité de transferts, dans la capitale même, entre différents types d'engagements. Car les placements qui s'offraient à la clientèle ne recevaient pas tous la même rémunération et leurs revenus ne s'échelonnaient pas sur des durées identiques. Il n'en fallait pas plus pour donner lieu à un jeu complexe entre les diverses formes de contrats. Dans le Paris d'Ancien Régime, elles étaient, comme ailleurs, au nombre de trois. Rentes ou obligations, perpétuelles ou viagères, chaque individu choisissait l'un ou l'autre de ces instruments d'épargne selon le rythme auquel il souhaitait recouvrer ses capitaux et percevoir ses revenus. Mais il modulait aussi sa décision en fonction de l'appréciation portée sur des questions plus générales comme l'évolution des prix ou la politique financière de l'État tant en matière de régulation que d'emprunt. Et comme, à un moment donné, l'État et les particuliers ne présentaient pas les mêmes risques, il s'ensuivait des déplacements entre placements publics et privés.

Ainsi, pendant la seconde moitié du XVIII ${ }^{c}$ siècle, la croissance du marché parisien a été secouée par la guerre de Sept ans, par la baisse réglementaire des taux entre 1766 et 1770 puis par la guerre de l'Indépendance américaine. L'activité de crédit a alors connu trois grandes phases : après une chute de 1755 à 1770, elle a rapidement repris pendant la décennie suivante avant de plafonner, voire de reculer à nouveau dans les années qui précèdent la Révolution. Mais parallèlement à ces mouvements globaux, le choix même des types d'acte utilisés a beaucoup évolué. Alors que la rente perpétuelle était au départ l'instrument dominant, à la veille de la Révolution, elle fait au mieux ${ }^{12}$ jeu égal avec les obligations dont la diffusion, entre temps, a été considérable (voir figure 3 ).

12. Le déclin des rentes perpétuelles est net quel que soit l'indicateur retenu (nombre d'actes, volumes et stocks). Comparée à l'évolution des obligations leur chute mesurée en volume est évidemment supérieure à celle qu'indiquent les stocks. 

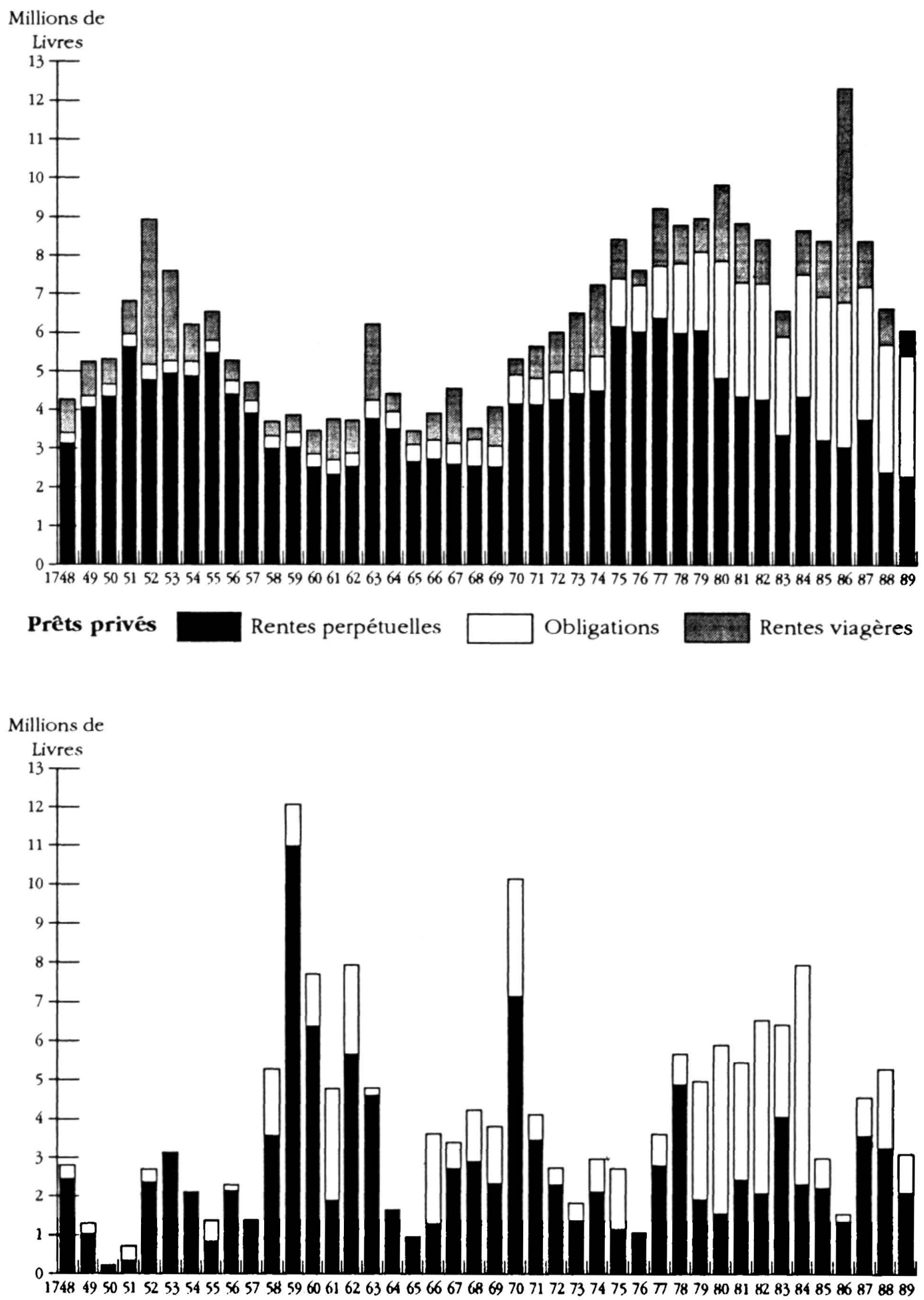

Rentes publiques Rentes perpétuelles Rentes viagères

FIG. 3. - Stocks des différents types de prêts de neuf études parisiennes, 1748-1789 
Cette substitution découle pour une large part de la concurrence entre crédit public et crédit privé, le premier étant en quelque sorte contraint à être plus novateur que le second. Les épargnants avaient appris d'expérience à douter des engagements de la couronne. Or, plus ses bailleurs de fonds potentiels avaient lieu de craindre qu'il ne se révèle un jour ou l'autre mauvais payeur, moins l'État pouvait s'en tenir aux conditions courantes. Il lui fallait alors payer des taux d'intérêt élevés sauf à trouver une clientèle qui ne prêtait pas dans le marché privé. En fait, il a joué sur les deux tableaux. L'importance même de ses besoins était d'ailleurs un atout. Contrairement à la plupart des emprunteurs, le Trésor bénéficiait de la loi des grands nombres pour ses appels de fonds et pouvait donc recourir aux rentes viagères, aux tontines et autres loteries sans prendre de risque supplémentaire. Il y trouvait même avantage puisqu'il attirait de la sorte des épargnants qu'il avait longtemps négligés comme les domestiques, les veuves et certains groupes de commerçants et de marchands particulièrement intéressés par ce genre de placement. L'élargissement de la clientèle avait cependant des limites et, tout en diversifiant toujours les produits qu'il proposait, l'État a donc continué à émettre des rentes perpétuelles ${ }^{13}$. Mais, comme ses innovations financières trouvaient aussi faveur auprès de ses prêteurs traditionnels, la couronne a dû relever les taux des perpétuelles en acceptant que celles-ci soient en partie payées en billets dépréciés ${ }^{14}$.

L'inventivité des finances publiques au cours du XvIrI ${ }^{e}$ siècle a pesé sur l'évolution du crédit privé car, à Paris, beaucoup de prêteurs répartissaient leur épargne entre les deux secteurs. Et, puisque le marché privé ne pouvait répondre à la concurrence de l'État en relevant ses taux du fait du plafond légal, il s'adaptait en changeant de type de contrat. C'est ce qui explique le succès croissant des obligations. Par rapport aux rentes perpétuelles, elles avaient l'avantage d'une durée définie tout en laissant une certaine latitude sur les taux pratiqués (qui toutefois ne pouvaient être explicitement spécifiés).

Le rééquilibrage entre les deux instruments s'est accentué à partir de la fin des années 1760 . La conjoncture y incitait parce que les épargnants eurent alors à se défendre contre une poussée d'inflation mais aussi parce qu'il leur fallut parer aux conséquences d'une décision publique. L'édit de 1766 avait réduit de 5 à $4 \%$ le taux plafond des rentes, ce qui revenait à

13. Par commodité, nous utilisons ici les termes de rentes viagères et de rentes perpétuelles de manière large. Nous désignons comme rentes viagères aussi bien les tontines que les rentes viagères stricto sensu. De même, à proprement parler, l'État n'emprunte plus directement en perpétuelles pendant la période considérée (les rentes émises portent sur des durées plus ou moins longues allant jusqu'à 32 ans). En revanche, les rentes émises sur le clergé, sur les États provinciaux, etc. sont encore des perpétuelles. C'est l'ensemble de ces emprunts que nous avons réunis sous le terme de rentes perpétuelles publiques. Ajoutons que la distinction public/privé elle-même est une simplification discutable (voir notamment infra, p. 83).

14. Voir, en particulier, F. VELDE et D. WEIR, «The Financial Market and Government Debt Policy in France, 1746-1793 », The Journal of Economic History, vol. 52, 1992, pp. 1-40. H. J. Shakespear, France, The Royal loans - Les emprunts royaux, 1689-1789, Shrewsbury, 1986. Le phénomène est d'ailleurs ancien et multiforme. Au XVI ${ }^{\mathrm{c}}$ siècle « le roi était souvent contraint d'accepter les monnaies à leur cours commercial quand il plaçait des rentes publiques auprès des villes » (M.T. Boyer-Xambeu, G. DelePlace, L. GILlard, Monnaie privée et pouvoir des princes, Paris, 1986, p. 373). 
rogner de $20 \%$ le produit des perpétuelles ${ }^{15}$. Cette perte amena des prêteurs qui avaient jusque-là placé leurs fonds en rentes à se reporter sur les obligations. Le changement introduit s'est d'ailleurs révélé durable. Après que le taux plafond eut été ramené au $5 \%$ traditionnel, les notaires ont continué à promouvoir les obligations au détriment des rentes perpétuelles. Pour les prêteurs, pareil choix avait une contre-partie. Substituer aux rentes perpétuelles des obligations de durée bien plus brève impliquait des coûts de transaction supérieurs. Pour atténuer l'inconvénient, les notaires et tout un monde d'intermédiaires qui évoluait dans leur mouvance ont alors diversifié leurs pratiques en offrant à leurs clients de se charger de la gestion quotidienne de leur portefeuille ${ }^{16}$. La croissance des obligations continua ainsi à être plus rapide que celle des rentes, y compris après qu'eût été reporté l'édit de 1766. Qui plus est, les très gros contrats, longtemps presque exclusivement en rentes perpétuelles, ont pris peu à peu la forme de l'obligation. L'instrument traditionnel du crédit s'est donc progressivement effacé. Entre le milieu du siècle et la dernière décennie de l'Ancien Régime, la valeur réelle des engagements en rentes perpétuelles a chuté de moitié alors que celle des obligations quintuplait.

L'utilisation des rentes viagères par les particuliers a suivi une évolution moins nette. Après s'être largement diffusés dans la première moitié du siècle ${ }^{17}$, ces prêts ont gardé une place relativement constante dans le marché privé en dépit de très fortes variations (de un à cinq entre deux années consécutives). Ils sont en fait restés la spécialité de très grands personnages (princes du sang, ducs et pairs, etc.) qui seuls étaient à même de tirer pleinement parti de cet instrument parce qu'ils en émettaient de très grandes quantités ${ }^{18}$. De ce point de vue, leur situation s'apparentait à celle de l'Etat qu'ils pouvaient donc suivre sur son terrain, le concurrençant ainsi directement. Mais il s'agissait d'un groupe trop restreint pour peser d'un grand poids sur le marché parisien.

Il reste qu'au delà de ces évolutions progressives, la forte présence de l'État sur le marché parisien se manifestait surtout par les brusques secousses qu'ont imposées les accroissements successifs de la dette publique —à la suite des guerres notamment ${ }^{19}$ - et dont l'irrégularité apparaît clairement sur la figure 3. Précisons qu'on n'y a fait figurer que les emprunts à moyen et long terme. Or ceux-ci exigeaient certains délais. Entre le moment où une émission était décidée et sa souscription effective il s'écoulait souvent une bonne année et parfois plus. Le recours aux emprunts à long

15. Cf., par exemple, J. GuYot, Répertoire universel et raisonné de jurisprudence civile, criminelle, canonique et bénéficiale, Paris, édition de 1784-1785, t. 9, article «intérêts ».

16. On en trouvera de nombreux exemples dans les dossiers que ces gestionnaires déposaient chez les notaires ou dans leurs propres archives (ainsi, AN, 26AP, papiers Pointard).

17. A en juger par le sondage effectué dans sept études (cf. supra note 10), les rentes viagères étaient encore peu utilisées au début du XVIII' ${ }^{\circ}$ siècle : entre 1730 et 1750 , elles représentaient $7 \%$ des prêts entre particuliers. Après une poussée rapide au milieu du siècle, leur progression se ralentit mais environ un prêt sur cinq est réalisé sous cette forme entre 1770 et 1789 .

18. Certaines années, les émissions de rentes viagères par de très grands personnages pouvaient représenter jusqu'au quart des sommes prêtées par notre échantillon de notaires.

19. L'effet des guerres sur le crédit est examiné dans un autre cadre par P. SERvaIs, La rente constituée dans le Ban de Herve au Xvilr siècle, Bruxelles, Crédit Communal de Belgique, Collection Histoire, $\mathrm{n}^{\circ} 62,1982$. 


\section{MODERNISATION DES SOCIÉTÉS TRADITIONNELLES}

terme n'était donc pas un moyen efficace pour lever des fonds rapidement. Néanmoins, la taille de ces emprunts a presque doublé entre la guerre de Sept ans et celle de l'Indépendance américaine.

De tels appels de fonds se faisaient-ils aux dépens du crédit privé ? On rappellera que, comparées aux engagements financiers globaux, les souscriptions de fonds publics sont alors restées relativement modestes de sorte qu'elles n'ont pu avoir de conséquences aussi considérables qu'en d'autres périodes ${ }^{20}$. Inversement, la capitale étant le centre d'émission des emprunts publics, si cette demande s'adressait aux seules ressources parisiennes, elle devait peser lourdement sur ce marché particulier. Mais si elle puisait dans l'épargne de la France entière, son impact sur le marché parisien pouvait être nettement plus faible. Pour élucider l'importance d'éventuels effets d'éviction sur le crédit dans la capitale, il faut donc considérer les liens entre les différents marchés, publics et privés, provinciaux et parisiens, à court terme et à long terme.

Dans le cas de l'Angleterre de la fin du XVIII ${ }^{e}$ et du début du XIX ${ }^{e}$ siècle, ce problème a fait depuis plusieurs années l'objet d'un débat actif. J. Williamson maintient que les emprunts massifs de la couronne britannique entre 1780 et 1815 ont retardé la révolution industrielle alors que J. Mokyr, C. Heim et $\mathrm{Ph}$. Mirowsky, et L. Neal soutiennent pour diverses raisons qu'ils ne provoquèrent pas d'effet d'éviction notable. Curieusement, la controverse est restée surtout insulaire ${ }^{21}$. En général, elle ignore donc l'autre protagoniste du conflit alors que la comparaison des deux pays serait pourtant instructive. Si l'on suppose qu'à un moment donné un même niveau de dépense militaire s'imposait aux deux belligérants, la France aurait dû en assumer plus aisément la charge, eu égard à la taille de son économie. En revanche, ses habitants étant plus pauvres, leur épargne moindre et les marchés financiers français moins développés, on pourrait penser que les ponctions de l'État dans l'épargne ainsi mobilisée aient eu des effets plus graves. D'autre part, l'abondance des informations autorise une analyse plus complète et plus probante du cas français puisqu'elle peut s'appuyer sur les variations du volume de l'activité financière, une donnée qui fait défaut outre-Manche où les notaires n'existent pas.

L'impact des demandes publiques sur le marché du crédit privé peut s'envisager de plusieurs façons. Si l'on raisonne sur les quantités, un effet d'éviction classique correspondrait à une corrélation négative entre le volume des emprunts publics et celui des emprunts privés à moyen et long terme. Si l'on s'attache aux prix, on tentera de mesurer l'influence d'une

20. La situation semble avoir été différente dans la seconde moitié du règne de Louis XIV. En 1700, par exemple, dans certaines études parisiennes les souscriptions d'emprunts publics dépassent et de loin l'activité de crédit privé qui se trouve ainsi très fortement réduite.

21. J. Williamson, "Why was British Growth so Slow During the Industrial Revolution? », The Journal of Economic History, Vol. 44, $\mathrm{n}^{\circ} 3$ (Sept. 1984), pp. 687-712; J. MOKYR, « Has the Industrial Revolution Been Crowded Out ? Some Reflections on Crafts and Williamson", Explorations in Economic History (3) 1987, pp. 239-319 ; C. HeIM and P. Mirowsky, "Interest Rates and Crowding Out during the British Industrial Revolution ", The Journal of Economic History, Vol. 47, (March 1987), pp. 117-139 ; L. NEAL, The Rise of Financial Capitalism. International Capital Market in the Age of Reason, Cambridge, Cambridge University Press. 1990. Voir aussi, sur la France, J. C. RILEY, The Seven Years War and the Old Regime in France: the Economic and Financial Toll, Princeton, Princeton University Press, 1986. 
hausse ou d'une baisse de l'intérêt payé par l'État sur l'activité du marché privé et sur les taux qui y étaient pratiqués. Dans le cas parisien, les informations nécessaires aux deux approches sont également disponibles. Nous connaissons en effet l'évolution mensuelle du volume du crédit à Paris pour les diverses formes d'emprunts, qu'ils soient privés (obligations, rentes viagères et perpétuelles) ou publics (rentes viagères et perpétuelles). Une réserve toutefois : les séries présentent au moins un biais. On a vu que lorsque l'État émettait des rentes perpétuelles, les bailleurs de fonds étaient souvent autorisés à régler leur souscription en effets publics à court terme ou en rentes plus ou moins dépréciés. En avril 1758, par exemple, il était prévu que les souscripteurs de rentes héréditaires à $4 \%$ payent moitié en argent comptant moitié en quittances de remboursement de rentes sur les aides et gabelles de juin 1720 . Ne portant que sur la valeur déclarée des emprunts, nos données peuvent donc exagérer les sommes en cause. Dans ce cas, l'impact d'un emprunt public d'un montant $\mathbf{M}$ devrait en fait être imputé à un appel de fonds $\mathbf{M}^{\prime}<\mathbf{M}$. En d'autres termes, nos sources risquent de sous-estimer l'effet d'éviction réel de certaines émissions en rentes perpétuelles. Les informations sur les taux soulèvent moins de difficultés ${ }^{22}$ puisque deux travaux récents apportent sur ce point des renseignements précis tant pour les fonds publics que pour le crédit privéés.

Sur cette base, si l'on examine d'abord le rôle des taux d'intérêt payés par l'État, on ne constate pas qu'ils aient eu quelque retombée que ce soit sur l'activité du crédit privé. Comme ils n'ont pas augmenté au moment d'emprunts massifs mais plutôt lors de crises financières, leurs fluctuations paraissent avoir reflété surtout des changements d'appréciation du marché sur l'aptitude de l'État à faire face à ses engagements (voir figure 4). Dans cette mesure, une hausse des taux publics tendait moins à réduire qu'à encourager les placements auprès des particuliers qui, aux yeux des investisseurs, devenaient alors des débiteurs bien préférables à l'État. A la limite, on pourrait même imaginer un effet d'éviction inverse. Mais l'analyse statistique $^{24}$ indique seulement que les variations des taux d'intérêt payés par l'Etat n'ont pas amené les investisseurs à modifier la composition de leur portefeuille de façon significative. Le marché privé a ignoré les changements dans l'appréciation des risques encourus par les prêts à l'État ${ }^{25}$. Une telle indifférence peut pourtant s'expliquer. Comme les taux publics flambaient lorsque les épargnants appréhendaient une banqueroute, le moment n'était

22. Voir pourtant infra, note 24 et note 36 .

23. Nous remercions F. VELDE et D. WEIR de nous avoir communiqué la série mensuelle de taux d'intérêt public qu'ils ont calculée à partir du cours de plusieurs titres émis par l'État. Nous empruntons d'autre part à T. M. LUCKETT la série comparable qu'il a établie pour les taux privés en étudiant l'escompte des lettres de changes ( Credit and Commercial Society in France, 1740-1789 », Ph.D. dissertation, Princeton University, 1992).

24. Notons que pour que l'analyse soit plus assurée il faudrait pouvoir confronter l'activité du crédit privé à un moment donné avec le taux d'intérêt des emprunts publics en cours de souscription. Nous disposons en fait d'une série où les taux d'intérêt sont ceux d'un groupe d'emprunts publics émis avant le milieu du siècle et qui donc ne reflètent le taux marginal que pour autant que les investisseurs voyaient les différents types de rentes publiques comme foncièrement équivalents.

25. Dans le cas anglais, la discussion des historiens ne s'est appuyée que sur cet examen des taux publics. 


\section{MODERNISATION DES SOCIÉTÉS TRADITIONNELLES}

certainement pas favorable à des échanges intenses et les transactions portant sur les fonds publics devaient se trouver pratiquement gelées. On comprendrait sinon difficilement, surtout après l'expérience de Law et la baisse forcée des taux dans les années 1766-1770, que les prêteurs n'aient pas envisagé qu'en cas de banqueroute l'État puisse être amené à une politique financière néfaste au crédit.

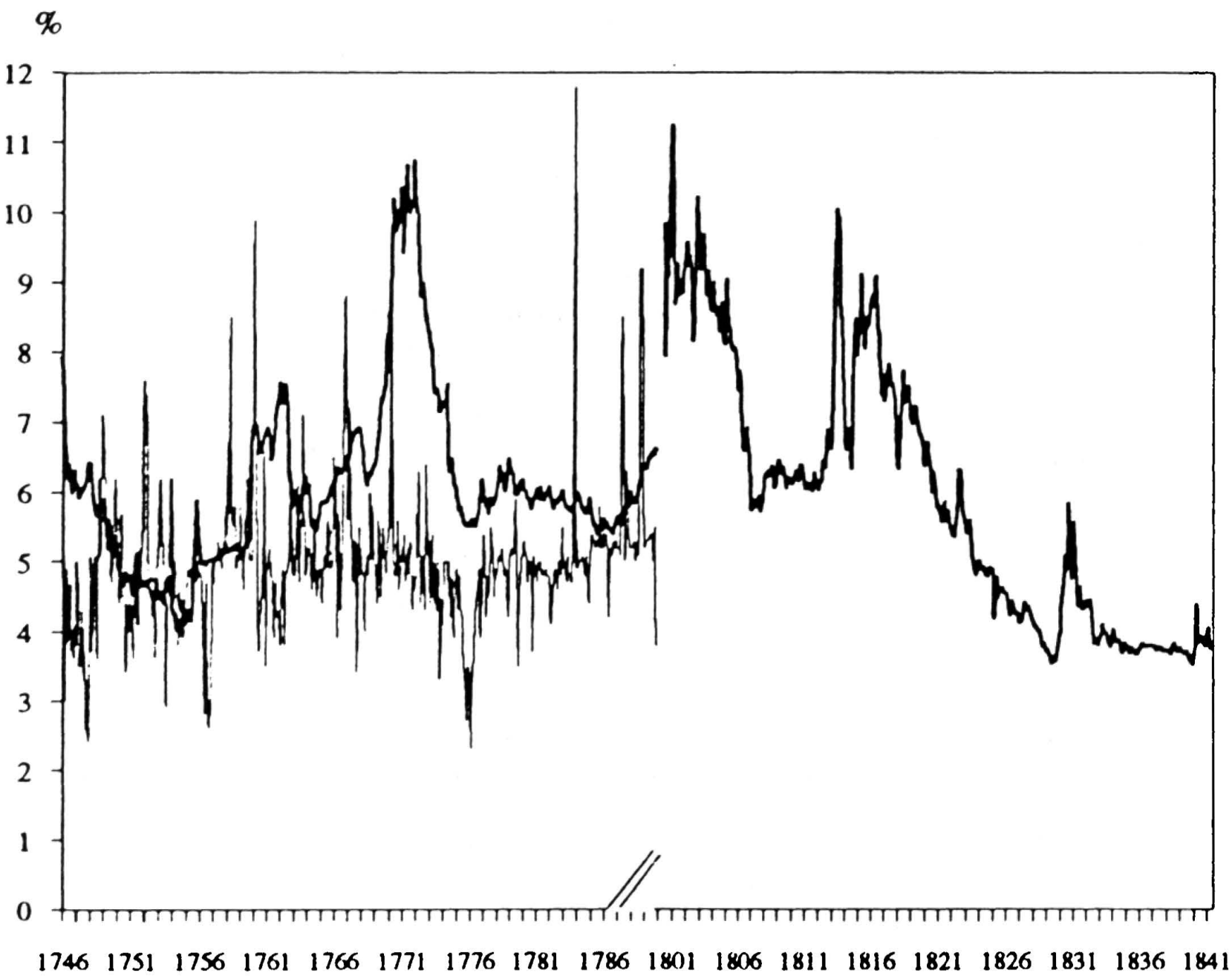

Court terme privé Fonds publics

Fig. 4. - Taux d'intérêt public et privé

Le constat est tout autre si l'on considère l'effet des emprunts d'État sur le volume du crédit privé. Nous nous en sommes tenus à une analyse statistique simple dont nous présentons ici deux variantes (cf. tableau 3). A vrai dire, quelle que soit la forme qu'on lui donne, elle suggère une même conclusion : les émissions publiques provoquaient un effet d'éviction non nul mais limité. Le mesurer précisément soulève pourtant plusieurs difficultés. Les multiples emprunts ont été d'importance très inégale (c'est pourquoi 
TABleau 3 - Les émissions publiques et le volume du crédit privé26

\begin{tabular}{|c|c|c|c|c|}
\hline \multirow{3}{*}{$\begin{array}{l}\text { Variable dépendante: } L P R I V \\
\text { Variables indépendantes: }\end{array}$} & \multicolumn{2}{|c|}{$\begin{array}{l}\text { Régression } \\
\text { principale }\end{array}$} & \multicolumn{2}{|c|}{$\begin{array}{l}\text { Régression } \\
\text { complémentaire }\end{array}$} \\
\hline & $\begin{array}{l}\text { Coefficient } \\
\text { estimé }\end{array}$ & $\begin{array}{c}t \text { de } \\
\text { Student }\end{array}$ & $\begin{array}{c}\text { Coefficient } \\
\text { estimé }\end{array}$ & $\begin{array}{l}t \text { de } \\
\text { Student }\end{array}$ \\
\hline & - & - & - & - \\
\hline Constante & 9,766 & 26,09 & 8,900 & 41,24 \\
\hline Temps & 0,0025 & 0,33 & $-0,004$ & $-0,64$ \\
\hline Temps $^{2}$ & 0,0009 & 5,55 & 0,001 & 6,54 \\
\hline Guerre & 0,286 & 9,13 & 0,209 & 6,87 \\
\hline \multicolumn{5}{|l|}{$\begin{array}{l}\text { Logarithme de la moyenne des souscrip- } \\
\text { tions mensuelles } \\
\text { de rentes perpétuelles publiques } \\
\text {. entre } 12 \text { et } 23 \text { mois avant le mois }\end{array}$} \\
\hline concerné & $-0,002$ & $-0,08$ & - & - \\
\hline . l'année qui précède le mois concerné & $-0,054$ & $-2,02$ & $-0,066$ & $-2,73$ \\
\hline $\begin{array}{l}\text { l'année qui suit le mois concerné } \\
\text { entre } 13 \text { et } 24 \text { mois après le mois }\end{array}$ & $-0,009$ & 0,30 & $-0,036$ & $-1,35$ \\
\hline $\begin{array}{l}\text { concerné } \\
\text { de rentes viagères publiques }\end{array}$ & $-0,138$ & $-4,65$ & - & - \\
\hline concerné & $-0,016$ & $-1,87$ & - & - \\
\hline l'année qui précède le mois concerné & $-0,004$ & $-0,52$ & $-0,002$ & $-0,18$ \\
\hline $\begin{array}{l}\text { l'année qui suit le mois concerné } \\
\text {. entre } 13 \text { et } 24 \text { mois après le mois }\end{array}$ & $-0,031$ & $-3,39$ & $-0,048$ & $-5,64$ \\
\hline concerné & $-0,041$ & $-4,01$ & - & - \\
\hline Nombre d'observations & 43 & & 45 & \\
\hline $\mathrm{R}^{2}$ & & & & \\
\hline $\mathrm{R}^{2}$ corrigé & & & & \\
\hline Somme des erreurs ${ }^{2}$ & & & & \\
\hline Ecart type de la régression & & & & \\
\hline Durbin-Watson & & & & \\
\hline Moyenne de LPRIV & & & & \\
\hline Indice de singularité & & & & \\
\hline
\end{tabular}

Variables : LPRIV $=$ logarithme du volume mensuel du crédit privé. Guerre $=$ variable muette : mois de guerre $=1,0$ sinon.

26. La méthode des moindres carrés ordinaires utilisée dans les tableaux 3,4 et 5 est susceptible de multiples critiques. Une première cause d'inquiétude tient, dans le tableau 3 , à la valeur élevée de l'indice de singularité pour la régression principale, signe de multicollinéarité. D'autre part, si la méthode a le mérite d'estimer des coefficients sans biais, elle bute sur des problèmes de non-stationnarité des erreurs et les t-statistiques sont probablement exagérés. Ces problèmes admettraient en principe une autre solution qui examinerait les différences de chaque variable entre la date $t$ et la date $t+1$ (en calculant celles-ci sur des périodes comme le trimestre ou le semestre car les données mensuelles ont des variations trop aléatoires). De telles régressions n'ont pas non plus une grande précision mais elles aboutissent à des résultats comparables à ceux qui sont présentés ici. On n’a pas reporté dans ces tableaux les coefficients de variables muettes pour les situations de chaque mois. 


\section{MODERNISATION DES SOCIÉTÉS TRADITIONNELLES}

nous exprimerons leur montant en logarithme). D'autre part, nos données d'origine étant mensuelles, nous avons naturellement cherché à tirer parti de ce degré de précision. Mais un découpage aussi fin s'est révélé mal adapté pour examiner les conséquences des appels de fonds publics sur le volume du crédit privé un mois donné. La durée d'ouverture des souscriptions était généralement brève, souvent un mois ou deux, voire quelques jours seulement. De ce fait, même lorsque l'État empruntait beaucoup, il pouvait s'écouler des mois pendant lesquels les notaires n'avaient pour ainsi dire aucun fonds public à placer. Une demande si variable devait donc soit faire appel à une épargne déjà constituée soit amputer pour quelque temps des sommes qui, sinon, auraient été sous peu disponibles pour le crédit privé. Il nous a semblé que des moyennes sur douze mois permettaient de mieux mesurer les effets de l'emprunt public en l'étalant quelque peu. Mais quel laps de temps prendre en compte? En aval, après la clôture d'une souscription, si les bailleurs de fonds venaient à manquer, c'était immédiatement, parce que leurs liquidités avaient été épuisées; ou bien un peu plus tard, quand ils avaient à rembourser un prêt à court terme souscrit pour l'occasion. Même dans ce cas, en raison de la brièveté de ce type de crédit, une période d'observation de deux ans paraît suffisante. Reste à considérer l'amont. Les épargnants étaient en effet à même de prendre leur décisison dès l'instant où l'emprunt était annoncé. Or le délai séparant l'édit qui le faisait connaître et la souscription effective était plus ou moins long. Souvent de l'ordre d'un an, il pouvait se réduire ou s'étendre. Certains emprunts étaient couverts en un semestre, les notaires recevant même parfois les fonds plusieurs mois avant qu'ils ne rédigent les contrats ${ }^{27}$. A l'inverse, après le lancement de la dixième tontine en novembre 1759 , il fallut un an pour que les notaires commencent à placer des contrats en grand nombre. Cette première vague fut suivie d'une seconde à l'automne 1761 , puis d'une troisième entre février et avril 1762, soit deux ans et demi après l'édit. Ainsi allonger la période d'observation dans le passé et dans le futur peut aider à mieux capter certaines situations. En même temps, la solution présente des inconvénients d'ordre statistique. Nous retiendrons donc deux approches. Dans une première régression nous inclurons des variables mesurant l'impact des emprunts publics avec des retards et des avances sur un et deux ans. Dans une régression complémentaire, nous ne rapporterons le montant des emprunts privés un mois donné qu'à la valeur moyenne des emprunts publics sur les douze mois précédents et les douze suivants.

Si l'on utilise les élasticités données par la première régression du tableau 3 pour estimer la réduction du crédit liée aux émissions publiques pendant la seconde moitié du XVIII ${ }^{\mathrm{e}}$ siècle, il apparaît qu'au total, quand l'État empruntait une livre, le crédit privé notarié parisien se contractait de huit sous environ. Cette estimation semble être un maximum. A en juger par la seconde régression, l'effet d'éviction ne serait que de quatre à cinq sous ${ }^{28}$.

27. Voir, par exemple, AN, Minutier Central, XII, 827, Emprunt de novembre 1777.

28. Les coefficients estimés dans le tableau 3 donnent des élasticités, soit la variation en pourcentage du crédit privé pour une variation de $1 \%$ du volume de chaque grand type d'émission publique. L'effet d'éviction s'en déduit : il suffit de multiplier ces élasticités par le rapport du volume des prêts privés sur celui de l'émission publique considérée. Le volume des prêts pri- 
Avec un effet d'éviction au plus de l'ordre de $40 \%$ et qui devait plutôt se situer autour de $20 \%$, nous sommes loin du un pour un théorique. L'État savait certes échelonner dans le temps la durée des souscriptions et il drainait des ressources provenant d'un éventail social et géographique beaucoup plus large que le crédit notarial parisien qui est le seul pris en compte par nos séries privées. D'une part, une fraction des sommes placées en rentes publiques étaient tirées du crédit à court terme, voire des bas de laines familiaux. Les émissions de viagères n'attiraient-elles pas la clientèle particulière des femmes veuves ou non mariées qui participait peu au crédit privé ? D'autre part, l'État avait un accès privilégié à des fonds étrangers, italiens et, plus encore, suisses et hollandais réunis par des intermédiaires parisiens ${ }^{29}$. Et Paris n'était qu'une fraction du marché financier français. Plus du tiers des prêteurs à l'État qui contractaient par le truchement des notaires de la capitale étaient des provinciaux ou des étrangers. Au reste, si la couronne plaçait l'essentiel de ses emprunts auprès de Parisiens, ceux-ci ne fournissaient pas nécessairement eux-mêmes la totalité des fonds. Et même si tel était le cas, leurs apports pouvaient fort bien ne pas avoir été prélevés dans leur portefeuille de prêts à des habitants de la capitale. On a vu que la petite minorité qui détenait le gros des rentes publiques possédait des actifs dispersés et pouvait donc attirer une partie de l'épargne provinciale à Paris. Par ailleurs, les inventaires après décès invitent à penser que ces élites étaient aussi actives dans le marché à court terme.

Les pratiques financières de l'État durant les guerres ${ }^{30}$ tendaient à émousser encore les effets d'éviction. Au début des conflits, la couronne empruntait peu à long terme, préférant lever les fonds nécessaires par des méthodes indirectes. Dans l'espoir d'une victoire rapide, elle requérait des fermiers généraux des avances plus considérables que de coutume ou retardait ses règlements en émettant des effets à court terme. Or fermiers généraux et fournisseurs de l'État n'avaient pas toujours les fonds propres qui leur étaient demandés et ils devaient donc emprunter chez les notaires. De ce fait, les conflits correspondent à des périodes de gonflement du crédit privé comme le montre la variable indicatrice «guerre». L'utilisation du crédit personnel de ces agents est symptomatique du monde financier d'Ancien Régime pour lequel la démarcation entre public et privé restait

vés est environ 3,2 fois supérieur aux émissions de viagères publiques et 2,7 fois celui des perpétuelles. Un coefficient de 0,01 correspond donc à un effet d'éviction de $3,2 \%$ pour les viagères et de $2,7 \%$ pour les perpétuelles. Pour chaque type d'acte, l'effet d'éviction total s'obtient en additionnant les sous-effets distingués. Ainsi, quand l'État empruntait une livre en perpétuelle, le crédit privé aurait baissé de 0,54 livre selon la première régression $((0,002+0,054+0,009+0,138) \times 2,7)$ ou de 0,27 selon la seconde $((0,066+0,036) \times 2,7)$. Pour les viagères, les résultats seraient respectivement de $0,29((0,016+0,004+0,031+0,041) \times 3,2)$ ou de 0,16 $((0,002+0,048) \times 3,2)$. L'effet global des émissions publiques $(42 \%$ pour la première régression, $22 \%$ pour la seconde) tient compte du poids respectif des viagères et des perpétuelles.

29. L'étude LXII traite avec des Suisses amenés par le banquier Mallet (sur ce cas voir, notamment, H. Luthy, La Banque protestante en France, Paris, Sevpen, 1961, t. II, p. 267 et C. Carrière, M. Courdurié, M. Gutsatz, R. Souarzony, Banque et capitalisme commercial, Marseille, Institut historique de Provence, 1976, p. 189) ; l'étude CXV multiplie les contrats passés avec des Hollandais réunis par un Le Couteulx ; l'étude XV a de même des relations privilégiées avec des bailleurs de fonds italiens, etc.

30. M. Morineau, "Budget de l'État et gestion des finances royales en France au XVIII" siècle ", Revue Historique, CCLXIV, 1980, pp. 289-336. 
floue. La vénalité des offices et l'emprunt forcé auprès des titulaires de fonctions publiques furent des moyens fiscaux largement employés car ils étaient rapides et sûrs ${ }^{31}$. Les ressources que l'on pouvait lever ainsi restaient toutefois limitées. Quand, les guerres durant plus que prévu, les dépenses militaires enflaient, il venait un moment où l'État devait résorber la masse de créances à court terme qui s'était accumulée et solliciter directement les épargnants. C'est alors seulement - quand la couronne se tournait vers le système notarial pour lever des fonds - que l'on voit fléchir l'activité du crédit privé. Il s'en suit d'ailleurs que les particuliers ne percevaient pas les guerres (qui, avec leur cortège d'emprunts, risquaient pourtant de rendre l'État insolvable) comme des événements menaçant réellement le système financier privé.

Les liens entre les deux marchés restaient d'autant plus limités que les épargnants ne pouvaient considérer emprunts privés et emprunts publics comme équivalents. Les prêts à l'État avaient des caractéristiques trop particulières. D'un côté les décisions du pouvoir étaient assez facilement observables; de l'autre, si diverses qu'elles aient été, les émissions publiques étaient entachées d'une même suspicion : elles auraient toutes été touchées en cas de banqueroute. A l'inverse, les particuliers en quête de crédit présentaient une grande variété de risques de sorte que quiconque souhaitait placer son épargne sur des débiteurs privés pouvait diversifier son portefeuille. Mais l'activité de ces débiteurs était beaucoup plus malaisée à connaître que celle de l'État. Dans cette mesure, certains épargnants préféraient les fonds publics, d'autres choisissaient de se constituer des portefeuilles mixtes. Selon le cas, l'effet d'éviction n'était pas le même.

Une fois choisie une forme de placement, il n'était pas toujours simple de transférer les fonds correspondants sur un autre instrument d'épargne. De telles opérations n'étaient certes pas impossibles et elles se sont d'ailleurs développées comme le montre la multiplication au cours du siècle des actes de transports de crédit. Mais le marché qui s'établissait ainsi n'en demeurait pas moins mal assuré. S'agissant de rentes publiques, outre le coût d'un transport, les prix étaient difficiles à établir puisque chaque emprunt présentait ses conditions et ses risques propres ${ }^{32}$. Le problème était un peu différent pour ceux qui avaient prêté à des particuliers mais le détenteur d'une rente perpétuelle privée qui souhaitait s'en défaire ne trouvait pas forcément preneur tant l'hétérogénéité des emprunteurs créait des risques de sélection adverse qui réduisaient la circulation. Les limites du second marché freinaient ainsi les possibilités de substitution entre différents types de placement. Dans ces conditions, l'emprunt public ne pouvait entrer en concurrence avec d'autres emplois que sur l'épargne récente dont le détenteur devait prendre en compte les difficultés de transfert dans le futur. Quiconque entendait réaliser un placement à long terme - que ce soit sur l'État

31. D. BIEN, «Les offices, les corps et le crédit d'État: l'utilisation des privilèges sous l'Ancien Régime ", Annales ESC, 43, $\mathrm{n}^{\circ} 2$, (mars-avril 1988) pp. 379-404.

32. En fait, certaines de ces valeurs faisaient l'objet d'une cotation régulière mais ce n'était pas le cas général. Cf. F. Bluche, Les magistrats du Parlement de Paris, Paris, Les Belles Lettres, 1960, p. 214 ; F. VeldE, D. WeIR, op. cit., pp. 12-13. 
ou sur des particuliers - avait donc intérêt à mettre jusque-là son épargne en prêts à court terme ${ }^{33}$.

Les responsables politiques ne pouvaient être indifférents au fait que les perpétuelles pesaient plus lourdement sur le marché privé que les viagères. Non pas tant parce qu'ils se souciaient d'encourager les transactions financières entre particuliers. Mais l'effet d'éviction posait un problème de finances publiques en ce qu'il mettait en évidence des possibilités de substitution entre divers placements. Si l'État voulait rapidement augmenter ses emprunts en perpétuelles, il s'engageait dans une concurrence onéreuse avec le marché privé. Concrètement, il devait relever le taux au dessus du $5 \%$

Tableau 4 - Les émissions publiques et les formes du crédit privé

Variable dépendante :

Variables indépendantes

Constante

Temps

Temps $^{2}$

Guerre

Baisse des taux

\begin{tabular}{cc}
\multicolumn{2}{c}{ L obligations } \\
Coefficient & $t$ de \\
estimé & Student \\
- & - \\
8,387 & 28,53 \\
0,017 & 2,35 \\
0,001 & 7,23 \\
0,329 & 8,23 \\
0,189 & 3,36
\end{tabular}

28,53

2,35

8,23

3,36
L perpétuelles privées

Coefficient $t$ de estimé Student

$\begin{array}{rr}11,225 & 30,22 \\ 0,042 & 4,67 \\ -0,001 & 3,50 \\ 0,399 & 7,90 \\ -0,231 & -3,26\end{array}$

Logarithme de la moyenne des souscriptions mensuelles des emprunts d'État :

. entre 12 et 23 mois avant le mois concerné

$\begin{array}{llll}-0,041 & -1,64 & -0,089 & -2,80 \\ -0,009 & -0,35 & -0,099 & -2,90 \\ -0,061 & -2,29 & -0,112 & -3,35 \\ -0,164 & -5,47 & -0,260 & -6,86\end{array}$

. l'année qui précède le mois concerné

. l'année qui suit le mois concerné

- entre 13 et 24 mois après le mois concerné

\begin{tabular}{rr}
\multicolumn{1}{l}{433} & \multicolumn{1}{c}{433} \\
0,85 & 0,42 \\
0,84 & 0,40 \\
33,45 & 53,42 \\
0,28 & 0,36 \\
1,70 & 1,56 \\
7,49 & \\
& 7,58 \\
71,72 & 71,72
\end{tabular}

Nombre d'observations

$\mathrm{R}^{2}$

$\mathrm{R}^{2}$ corrigé

Somme des erreurs ${ }^{2}$

Ecart type de la régression

Durbin-Watson

Moyenne de L obligations

Moyenne de L perpétuelles privées

Indice de singularité

sion

Variables : $\mathrm{L}$ obligations = logarithme du volume mensuel des obligations. $\mathrm{L}$ perpétuelles $=$ logarithme du volume mensuel des rentes perpétuelles privées. Baisse des taux $=$ variable muette : mois où le denier légal était 25 au lieu de $20=1,0$ sinon. Pour les autres variables voir supra.

33. Là aussi, les notaires pouvaient offrir leurs services. Recevant régulièrement des sommes à placer, ils étaient à même de les engager à court terme jusqu'à ce qu'ils trouvent un emprunteur qui convienne à chacun de leurs bailleurs de fonds. Cf. J.-M. MORICEAU, G. POSTELVINAY, Ferme, entreprise, famille, Paris, Éd. de l'EHESS, 1992, p. 55 et note 125. 


\section{MODERNISATION DES SOCIÉTÉS TRADITIONNELLES}

courant en acceptant de recevoir une partie du paiement en papiers à court terme ou en anciennes rentes dépréciées. Émettre des viagères permettait à l'État de placer ses emprunts en se confrontant moins directement au marché privé. Mais, si cette stratégie s'est affirmée pendant la seconde moitié du $X{ }^{\prime} I_{I}{ }^{c}$ siècle, les émissions de rentes perpétuelles publiques n'ont jamais été complètement abandonnées, ce qui s'explique sans doute en partie par le fait que ce type d'acte restait recherché par nombre de prêteurs qui épargnaient dans un but dynastique.

Quand l'offre globale de crédit privé se réduisait suite à des émissions publiques, les instruments de crédit les moins flexibles souffraient plus que d'autres. Comme le montrent les régressions du tableau 4, les rentes perpétuelles étaient particulièrement affectées par un gonflement du crédit public. Chaque livre ajoutée à la dette de l'État réduisait leur volume de près de 7 sous alors que l'effet d'éviction sur les obligations était presque moitié moindre ${ }^{34}$. Les réalisations de rentes perpétuelles ont également fléchi suite à la baisse réglementaire des taux entre 1766 et 1770 quand, dans le même temps, les obligations ont au contraire augmenté. D'autre part, ces dernières subissaient le contre-coup d'une émission publique pendant une période plus courte. Ces réactions distinctes ou opposées à la politique de baisse des taux et aux emprunts d'État relèvent d'une même explication. Dans les deux cas, l'offre dans le marché privé s'est trouvé réduite, soit parce que les rémunérations avaient baissé soit parce que d'autres investissements attiraient l'argent des prêteurs. Habituellement négociées à $5 \%$, les perpétuelles ont pâti du changement de réglementation de 1766 car elles ne permettaient pas de parade à la décision gouvernementale. En revanche, en utilisant l'obligation qui ne mentionnait pas l'intérêt, il était loisible aux parties de s'accorder pour ajuster les taux aux conditions du marché. De ce fait, ce type de contrat n'était pas atteint par un édit abaissant le denier légal et pouvait aussi concurrencer les emprunts d'État ce qui réduisait les effet d'éviction.

La chute des obligations devançait nettement les réalisations d'emprunts publics ce qui indique que l'on retirait assez tôt les sommes qui devaient être placées en souscription publique. De plus, comme tous les coefficients de la variable « emprunts d'État » sont négatifs, il semble que, pendant la seconde moitié du XVIII ${ }^{\mathrm{e}}$ siècle, l'obligation n'était plus utilisée comme un instrument d'attente mais était majoritairement devenue un engagement de moyen terme. Il est donc plus probable qu'avant d'avoir trouvé un placement définitif pour leur argent, les prêteurs soit le thésaurisaient soit le prêtaient en billets à court terme. Or ce genre d'épargne anticipant un appel public réduisait les phénomènes d'éviction qui ne devaient apparaître que si l'emprunt excédait les prévisions des épargnants.

Plus généralement, toute épargne préalable avait à arbitrer entre court et long terme. Ainsi des prêteurs à court terme comme les marchands et les

34. La somme des élasticités données au tableau 4 est de 0,56 pour les premières et de 0,27 pour les secondes. Le rapport des unes et des autres aux émissions publiques étant respectivement de 0,60 et de 0,66 , l'effet global d'éviction serait de $0,56 \times 0,60=33 \%$ pour les rentes (soit 7 sous par livre) et de $0,27 \times 0,66=18 \%$ pour les obligations (soit moins de 4 sous par livre). 
bourgeois souscrivaient une importante fraction des emprunts publics ${ }^{35}$. On sait peu de choses des variations d'activité du vaste marché du crédit à court terme dont ne sont connus avec précision que les taux qui y étaient pratiqués $^{36}$. Mais il est clair que le niveau de ces derniers était lié au volume des affaires privées (cf. tableau 5). La relation jouait dans le temps, aussi bien par avance qu'avec retard. Quand les taux du court terme se tendaient, les volumes se réduisaient de façon durable. Ainsi les «famines d'argent » qui venaient régulièrement perturber les circuits financiers du court terme ${ }^{37}$ rejaillissaient-elles également sur ceux du moyen et long terme. L'interprétation des coefficients d'avance est plus complexe car il est peu probable que des engagements longs aient été affectés par des anticipations portant sur les taux courts. Un prêteur qui avait trouvé un emprunteur sûr n'aurait probablement pas tenté de remettre en question l'échange de capital convenu lorsqu'il pressentait un relèvement de ces taux car il lui en aurait coûté par la suite de retrouver un débiteur fiable. Mieux vaut donc lire les coefficients correspondants à l'envers comme l'indication que les taux d'intérêt étaient

Tableau 5 - Volume d'activité du crédit privé et taux d'intérêt

Variable dépendante : LPRIV

variables indépendantes

Constante

Temps

Temps $^{2}$

Guerre

Baisse des taux

\section{Coefficient estimé}

11,362

- 0,041

0,002

0,068

$-0,017$ $t$ de

Student

30,98

6,15

11,01

2,25

- 0,33

Logarithme de la moyenne des taux

d'intérêt à court terme

. entre 12 et 23 mois avant le mois concerné

$-0,503$

$-0,234$

- 0,558

$-0,631$
- 3,81

- 1,72

- 4,07

$-4,95$

. l'année qui suit le mois concerné 13 et 24 mois après le mois concerné

Nombre d'observations

$\mathrm{R}^{2}$

$\mathrm{R}^{2}$ corrigé

Somme des erreurs ${ }^{2}$

Ecart type de la régression

Durbin-Watson

Moyenne de LPRIV

Indice de singularité

434

0,69

0,68

31,18

0,27

1,38

8,43

88,30

35. Ph. Hoffman, G. Postel-Vinay, J.-L. Rosenthal, article cité.

36. Cf. T. M. LuCKETT, op. cit. Soulignons toutefois que ce travail ne fournit pas les taux d'intérêt payés sur des lettres de change circulant à l'intérieur du pays mais ceux de lettres entre Paris et Londres. Nous avons pourtant admis que ces derniers pouvaient être pris comme indicateur des taux à court terme du marché parisien.

37. Cf. T. M. LUCKETT, op. cit. 


\section{MODERNISATION DES SOCIÉTÉS TRADITIONNELLES}

sensibles aux variations des volumes échangés. Il est en effet vraisemblable que certaines famines d'argent aient résulté de crises propres au marché du crédit notarié puisque les banquiers - gros émetteurs de lettres de change - puisaient couramment des ressources auprès des notaires ${ }^{38}$.

En dépit de liens étroits, l'intégration entre marché privé et marché public restait limitée. Cette situation tient à la structure du marché qui était loin d'être toujours favorable à la liquidité des placements et à leur dispersion dans l'espace. Ce que l'on sait des fortunes des riches Toulousains, des officiers du bureau des finances de Lille ou des magistrats du Parlement de Paris au XVIII ${ }^{\mathrm{e}}$ siècle montre qu'une fraction seulement de leurs actifs financiers était d'origine extra-régionale ${ }^{39}$. Dans le cas des rentes publiques en particulier, la répartition géographique de leurs souscripteurs était tributaire des clientèles qu'avaient su se constituer les intermédiaires qui en assuraient le placement. Ainsi, parmi les notaires de la capitale, les uns s'adressaient presque exclusivement à des Parisiens, d'autres sollicitaient des bailleurs de fonds hollandais ou gascons, suisses ou béarnais, italiens ou normands. Au reste, ce circuit privé répondit assez bien à la tâche. En tout cas, même si les affaires publiques représentaient une large part de ses activités, il demeurait indépendant de l'administration royale qui se satisfaisait du rôle qu'il jouait et ne tenta pas de placer et de payer elle-même ses rentes à travers le pays. Cette politique s'explique par la situation singulière de la place de Paris et de l'organisation du marché notarial. D'une part la capitale était exceptionnellement riche et les ressources y étaient très concentrées alors que les prêteurs provinciaux étaient plus dispersés et disposaient de moins de fonds. Il était donc compréhensible que l'Etat se soit appuyé sur le notariat parisien. D'autre part, bien que l'intégration financière restât partielle, les réseaux tissés par les études de la capitale étaient assez ouverts pour mobiliser des capitaux et limiter les effets d'éviction à quelque $20 \%$. L'État ne collectera directement des fonds en province qu'après la Révolution, quand les circuits traditionnels auront été affaiblis et qu'une réforme administrative générale aura réduit les coûts de l'opération. Comme enfin les demandes publiques sont restées relativement faibles au XVIII ${ }^{\mathrm{e}}$ siècle - au moins jusqu'à la guerre d'Amérique - le circuit parisien a suffi à approvisioner l'Etat. Nous rejoignons ainsi les conclusions de $\mathrm{F}$. Velde et $\mathrm{D}$. Weir pour lesquels la crise de ressources qui minait l'État à la veille de la Révolution n'était pas imputable au système financier public mais à l'insuffisance des revenus fiscaux.

Le système financier privé, quant à lui, a conservé son fonctionnement inter-individuel - avec les aléas inhérents à une telle structure - et des coûts de transaction sans doute élevés. Il ne s'en est pas moins révélé capable de mobiliser des sommes considérables et croissantes provenant d'un large éventail de bailleurs de fonds. Dans les années 1780, les RohanGuéménée ou les Orléans lèvent chacun plus d'un million de livres en rentes

38. Reste alors à analyser quels facteurs déterminaient les variations de taux et de volumes en dehors des emprunts de l'Etat.

39. Cf. J. Sentou, Fortunes et groupes sociaux à Toulouse sous la Révolution, Toulouse, Privat, 1969; Ph. Rosset, Les officiers du bureau des finances de Lille, 1691-1790, Genève, Droz, 1991, pp. 152-156; F. BluCHE, op. cit., pp. 212-216. 
- des viagères surtout - auprès de plusieurs centaines d'épargnants. D'autres empruntent des sommes similaires en quelques contrats d'obligation. Si ces affaires frappent par leur importance, il ne faut pas en conclure que le crédit notarial n'alimentait que les dépenses somptuaires de quelques aristocrates. Outre que ces familles investissaient dans des secteurs assez divers, une foule d'emprunteurs plus anonymes et plus directement liés à l'activité économique se pressait derrière les grands noms. Quelles qu'aient été ses limites, le marché parisien avec ses multiples ramifications n'apparaît ainsi ni inefficace ni figé, qu'on se place du point de vue des particuliers ou de l'État. Qui plus est, la pression exercée par les demandes publiques, en même temps qu'elle le déstabilisait, l'a étendu et diversifié et, le faisant évoluer, l'a finalement consolidé.

\section{Le marché du crédit et la Révolution}

Mais ce circuit complexe et fragile fut particulièrement vulnérable au choc provoqué par l'événement révolutionnaire de sorte qu'à l'extrême fin du siècle, son activité était presque réduite à néant. Et si une série de réformes a alors posé les bases d'un redressement, il ne retrouva plus jamais sa splendeur passée, à Paris tout au moins ${ }^{40}$.

En 1799, trois ans après la disparition des assignats, les prêts notariés entre particuliers n'étaient ainsi que le quart (en volume) ou le dixième (en stocks) de ce qu'ils avaient été dans les années 1780. Les principales mesures de redistribution des richesses (l'abolition de la vénalité des offices, la nationalisation des biens d'église, l'émigration...) ne pouvaient qu'affaiblir, pour un temps au moins, les échanges de capitaux privés, à la fois parce qu'elles réduisaient les revenus de certains clients importants des réseaux notariaux et parce qu'elles remettaient en cause la valeur de l'information qu'ils détenaient.

Mais la décision d'émettre des assignats devait avoir des conséquences beaucoup plus graves et durables. L'activité d'un système de crédit à taux fixes soumis à une forte inflation tend normalement à baisser. Or, à Paris, la période des assignats fut d'autant plus dévastatrice que les particuliers détenaient une grande partie de leurs actifs financiers sous la forme de rentes ${ }^{41}$. Ces contrats qui interdisaient au bailleur de fonds de réviser les taux d'intérêts ou d'agir sur la durée des rentes étaient le plus mauvais placement dans ces années d'inflation rapide. Alors que leur capital, intérêt et principal, fon-

40. Le crédit public subit lui aussi les conséquences de la Révolution puisqu'il n'y eut pas d'emprunt public important avant 1817. Cf. M. MARION, Histoire financière de la France depuis 1715, 6 volumes, Paris, 1914-1931. M. D. Bordo, E. N. WhITE, " A tale of Two Currencies: British and French Finance During the Napoleonic Wars ", The Journal of Economic History, Vol. 51, no 2 (June 1991), pp. 303-316.

41. Celles-ci représentaient $62 \%$ des stocks de dettes privées dans les années 1780 . Or, en se référant aux stocks et non aux encours, on sous-estime la part des rentes dans les portefeuilles d'épargnants parisiens. A cette date, en effet, les rentes avaient perdu de leur ancienne importance, ce qui transparaît, bien sûr, dans la valeur des stocks (soit les engagements de l'année pondérés par leurs durées). Mais les affaires anciennes toujours en cours contenaient une plus forte proportion de rentes. 
dait avec la hausse des prix, les prêteurs ne pouvaient ni en demander un retour immédiat ni en refuser le remboursement dans l'espoir d'un rétablissement d'une monnaie-argent. Au contraire, les emprunteurs étaient libres de s'acquitter quand bon leur semblait en assignats dévalués. Avec l'accélération de l'inflation, le système financier a donc organisé un vaste processus de redistribution de créditeur à emprunteur. La leçon fut entendue. Naturellement, peu d'épargnants se fièrent ensuite à des contrats de rentes pour placer leurs capitaux. Mais surtout nombre d'entre eux se tinrent durablement à l'écart du marché financier. D'autant que, même après Brumaire, beaucoup ont continué à craindre que, à la suite d'une crise de régime, l'État ne retourne à des pratiques financières néfastes au crédit privé. Et l'histoire politique mouvementée de la première moitié du XIX ${ }^{e}$ siècle n'était pas tout à fait de nature à les rassurer.

Pourtant, le système financier s'est lentement reconstruit après la Révolution en s'appuyant sur des structures nouvelles et considérablement simplifiées. Les emprunts publics se sont fondus dans la rente consolidée. D'autre part, en légalisant le prêt à intérêt dont le taux pouvait désormais être stipulé dans tous les contrats, la Révolution rendait inutile le recours aux rentes ce qui acheva de rendre l'obligation l'instrument courant du crédit notarié ${ }^{42}$. Si Napoléon réintroduisit en 1807 le plafond traditionnel de $5 \%$ pour les emprunts hypothécaires, la légalité des contrats qui stipulaient les taux et les délais ne fut pas remise en question. La réforme du système hypothécaire de l'an VII fit enfin mieux connaître les garanties offertes en mettant en place une administration décentralisée qui assura la publicité des engagements hypothécaires même si l'information disponible demeurait à la fois partielle et relativement coûteuse.

La reprise du crédit notarié à Paris ne s'en est pas moins fait attendre. Au début des années 1840 , le stock de prêts n'avait toujours pas retrouvé le niveau qui était le sien sous l'Ancien Régime. Ce déclin s'explique d'abord par le brutal raccourcissement des engagements après la Révolution. La durée moyenne des prêts qui atteignait près de dix ans dans les années 1780 n'était plus que de deux ans en 1798. De même, la remontée ultérieure des stocks reflète pour une part la lente augmentation des durées. Après avoir fléchi à la fin de l'Empire, celles-ci s'allongent à nouveau sous la Restauration. Cette tendance a d'ailleurs connu des hauts et des bas. Quand le crédit se contractait, c'était principalement par un jeu sur les durées, et pas seulement de façon préventive. Après une crise, on n'assistait pas à une multiplication des prorogations et les obligations qui avaient été passées pour un

42. Cf. P. Sagnac, La législation civile de la Révolution Française, Paris, 1898, p. 204. Le contrat de rente perpétuelle «était très usité avant la loi du mois d'octobre 1789 qui fit cesser la prohibition du prêt à intérêt ; depuis, on n'en voit presque plus entre particuliers. La raison en est simple : lorsqu'en prêtant son argent pour un temps limité, on peut en retirer le même intérêt pendant le temps du prêt qu'en aliénant moyennant une rente, on trouve plus commode de recourir au premier moyen qui nous laisse le droit de rentrer dans la possession de notre capital au bout du temps convenu... si bon nous semble. Le contrat de constitution, au contraire, qui donne au débiteur, comme le contrat de prêt le droit de se libérer quand il veut, ôte au créancier le droit d'exiger le remboursement de son capital ; en sorte que, sous ce rapport, le désavantage est du côté du créancier ». A. J. MASSÉ, Le parfait notaire ou la science des notaires, $6^{c}$ éd. Paris, 1827, t. 2 , pp. $72-73$. 
temps bref n'en étaient pas moins quittancées rapidement. Entre la campagne de Russie et la seconde Restauration, par exemple, plus que leur nombre et que leur montant, c'est la durée des contrats qui est modifiée : pendant ces quelques années la baisse est de l'ordre du quart (elle est de près d'un an). Ces pratiques nouvelles permettaient une adaptation souple aux incertitudes politiques et évitaient aux prêteurs de s'exposer pour des échéances lointaines jugées trop aléatoires (même si l'assurance qu'on y gagnait n'était pas gratuite, la rotation rapide des crédits imposant des coûts de transactions supérieurs à ceux d'avant 1789).

Malgré tout, la remontée du crédit privé s'est essoufflée. Le stock des prêts plafonne après un maximum en 1826 parce que, passé cette date, la durée moyenne des prêts se stabilise autour de cinq années alors que le volume des transactions stagne ou même décline pour nombre d'études pendant les années trente. A en juger par l'évolution des neuf études que nous avons suivies en détail, le crédit après 1815 suivrait une courbe en cloche : après une croissance régulière jusqu'en 1826 , leur activité connait un plateau avant de chuter à partir de 1832 . Ce recul reste toutefois mal assuré parce qu'il ne repose que sur quelques études et qu'il s'accompagne d'un bouleversement de leur hiérarchie, un fait sans précédent jusque-là. Certaines des principales études du XVIII ${ }^{\mathrm{s}}$ siècle ne traitent alors presque plus d'opérations de crédit. D'autres qui avaient longtemps vivoté se développent au point que l'une d'entre elles devient la plus importante de notre sondage ${ }^{43}$. Quelles que soient ses limites, cet échantillon fait donc au moins apparaître le redéploiement en cours au sein du notariat parisien. Sous la monarchie de Juillet, alors qu'un groupe tente avec succès de se réorienter, d'autres perdent peu à peu l'essentiel de leur activité de crédit, ce qui semble souvent lié au tarissement de leur ancienne clientèle aristocratique.

Pour saisir les tendances globales du marché, on préférera cependant une base d'information mieux assise. Deux coupes en 1820 et 1840 portant sur un plus grand nombre d'études conduisent ainsi à un bilan moins négatif. Elles indiquent non plus un recul mais une stagnation du crédit notarial entre ces deux dates. Mais là aussi le groupe est loin d'être homogène. Si le niveau d'activité de l'ensemble se maintient peu ou prou c'est parce qu'il est soutenu par la forte croissance d'une minorité d'études. En 1840, une grande partie du crédit se concentre ainsi chez quelques notaires réalisant chaque année un total de prêts dépassant le million et demi de francs. Les écarts traditionnels entre les études se sont considérablement creusés. Chez beaucoup, les transactions sont devenues irrégulières et ne portent que sur des montants limités ; d'autres traitent de très gros contrats et tout spécialement ceux d'entre eux qui ont su adosser leur activité à celle des banques ${ }^{44}$.

Cette participation de banquiers à des affaires de moyen ou long terme conduit à revoir l'image habituellement donnée de la situation financière à l'époque. On oppose classiquement les établissements de crédit censés s'être

43. Il s'agit de l'étude IX. A l'inverse, une étude autrefois considérable comme l'étude CXV perd alors rapidement une grande part de son activité. Le déclin s'accélère d'ailleurs lorsque l'étude change de mains dans les années 1830.

44. Tel est le cas, par exemple, de l'étude XV' (Caisse des dépôts), de l'étude XX (Laffitte), de l'étude LXII (Mallet), etc. 


\section{MODERNISATION DES SOCIÉTÉS TRADITIONNELLES}

cantonnés à l'escompte ou aux prêts publics et les réseaux notariaux spécialisés dans les crédits longs. Le cas parisien amène plutôt à souligner les liens étroits entre le notariat et la banque. Des banquiers empruntent et prêtent dans des études notariales à des industriels comme à des aristocrates, à des promoteurs immobiliers comme à des entrepreneurs agricoles. En un sens, le fait n'est pas nouveau puisqu'au XVIII ${ }^{\mathrm{e}}$ siècle nombre de banquiers collaboraient intimement avec certaines études. Mais les rapports ne sont plus les mêmes. Désormais, dans les contrats où figurent un banquier, le notaire n'intervient plus que comme greffier et semble avoir perdu son rôle traditionnel d'intermédiaire. En témoigne la diffusion de prêts sous forme d'ouvertures de crédit. Ce type d'engagement hybride est souvent notarié mais l'argent s'échange en dehors de l'étude. Même là où le crédit notarié s'est renforcé entre 1820 et 1840 , il n'est pas dit pour autant que les notaires aient été les moteurs de cette expansion, et il est sûr que les banquiers y ont joué un rôle important.

Les relations qui s'instaurent entre notaires et banquiers sont de complémentarité et de coopération. Elles sont aussi de concurrence. Mais dans les deux cas, on ne saurait rendre compte du renforcement du rôle des banquiers à l'époque par les économies de taille que la plus grande spécialisation de ces intermédiaires permettait de réaliser. L'argument est en effet trop général pour expliquer la chronologie que la conjoncture particulière du marché parisien paraît au contraire aider à mieux comprendre. Au $\mathrm{XVIII}^{\mathrm{c}}$ siècle, puisque deux des trois types de contrats existants ne leur permettaient pas de savoir le temps pour lequel ils s'engageaient, les prêteurs multipliaient les (relativement) petits prêts pour amortir les risques. A l'inverse, après la Révolution, la généralisation d'un instrument de prêt à moyen terme réduisait les aléas dus au temps mais augmentait les coûts de transaction. Pour atténuer l'inconvénient, il fallait accroître la taille des prêts quitte à être particulièrement vigilant sur le choix des emprunteurs.

La situation tendait ainsi à écarter la petite clientèle des réseaux notariaux. De plus, ceux qui détenaient de faibles sommes pouvaient préférer les banques dont l'organisation répartissait les risques encourus sur l'ensemble des ressources qu'elles prêtaient au lieu d'enfermer l'épargnant dans un tête à tête toujours incertain avec un emprunteur unique comme le faisait le crédit notarial. En tout cas, force est de constater que la distribution des prêts passés dans les études a alors glissé vers le haut mais s'est trouvée tronquée vers le bas. Les notaires ne traitent plus avec les mêmes bailleurs de fonds que par le passé. Les petits prêteurs se font rares, et les petits emprunteurs tendent à disparaître. Parmi les moins grosses affaires (qui généralement portent malgré tout sur des montants allant de 1000 à 5000 francs), beaucoup s'intègrent dans les montages financiers que réalisent les notaires pour réunir les sommes demandées par un client important disposant d'excellentes garanties.

La masse des artisans aisés, des commerçants et des multiples petits rentiers qui avaient autrefois apporté leur épargne aux réseaux notariaux les ont désertés. Avant la Révolution, ils y intervenaient surtout comme prêteurs et, à ce titre, ils ont peut-être particulièrement souffert de la redistribution liée aux assignats. Mais il est peu probable que quarante ou cinquante ans plus 
tard leur épargne en soit encore affectée et que leur besoin de financement se soit tari. Bien que le problème soit mal connu, il faut plutôt supposer que cette clientèle s'est reportée vers d'autres circuits de placement et de crédit.

L'État qui à partir de la Restauration emprunte à nouveau après une absence d'un quart de siècle aurait-il été un autre concurrent du circuit notarié ? Il est en tout cas certain que les rentes publiques deviennent alors au moins aussi sûres et liquides que les prêts aux particuliers. Cette nouvelle donne entre crédit privé et crédit public pourrait avoir amené les petits épargnants à concentrer leurs capitaux dans les fonds d'État en sorte que seuls les riches auraient continué à participer au marché financier privé. Mais l'analyse des liens entre marché public et marché privé ne peut plus être aussi détaillée qu'au siècle précédent car l'information s'appauvrit au $\mathrm{XIX}^{\mathrm{e}}$ siècle. Ayant dès lors un circuit propre, les émissions publiques échappent à l'observatoire que fournissaient les archives notariales, ce qui empêche de comparer les clientèles. De même, les sources notariales permettent toujours de reconstituer l'évolution de l'activité du crédit privé, non plus celle du crédit public. A défaut, on devra donc raisonner ici indirectement sur les excédents et déficits publics annuels qui ne sont d'ailleurs connus qu'après $1815^{45}$. D'autre part, la seule série de taux d'intérêt disponible est celle des rentes d'État ${ }^{46}$. L'examen de ces informations réduites amène toutefois à deux conclusions. La première prolonge celles déjà tirées pour le siècle précédent ; l'autre au contraire s'en écarte.

Ainsi, l'activité du crédit notarial ne répond toujours pas aux variations des taux d'intérêt publics. Après la banqueroute des deux tiers, l'État a longtemps été absent du marché et n'a que lentement rétabli sa réputation de bon payeur. Les taux qu'il versait se sont alors réduits peu à peu suivant une tendance longue à la baisse, mis à part de brusques à-coups créés par des conjonctures politiques instables (voir figure 4). Leurs variations n'étaient donc pas liées à l'évolution de la dette nationale, mais, comme par le passé, à des changements dans les appréciations des risques encourus. Et il n'y avait pas plus de raison qu'au siècle précédent pour que les taux d'intérêt payés par l'État aient une quelconque influence sur le marché privé.

En revanche, l'analyse ne détecte plus la relation relevée précédemment entre le flux des prêts de particulier à particulier et les besoins financiers de l'État. Positifs ou négatifs, les exercices budgétaires n'ont aucun impact statistique sur les volumes engagés dans le marché privé. Il est vrai qu'en matière d'emprunt, l'État avait profondément modifié sa politique. De 1798 à 1841, il ne procéda qu'à deux émissions importantes : au début de la Restauration pour régler les indemnités aux coalisés, et de 1825 à 1827 pour le Milliard des émigrés. Dans le premier cas, l'émission aurait pu peser sur le crédit privé d'autant que l'argent fut dépensé à l'étranger ${ }^{47}$. Mais l'appel de fonds est intervenu après plusieurs années de très faible activité financière et

45. L. Fontvieille, Evolution et croissance de l'État francais de 1815 à 1969, 1976, Cahier de l'ISMEA, série AF, no 13, Paris, 1976, p. 1937.

46. F. VELDE et D. WEIR, op. cit.

47. C'était pour partie le cas au XviII' siècle, cf. M. Morineau, op. cit., p. 307, note 54. 
de thésaurisation, notamment pendant les Cent Jours, de sorte que les prêteurs ont pu répondre simultanément aux demandes publiques et privées. La situation était tout autre au moment du Milliard des émigrés. Mais l'emprunt était destiné à une vaste opération de redistribution au profit de personnes fortunées. Aussi beaucoup de ceux qui en ont bénéficié, de riches aristocrates en particulier, ont-ils rapidement rééquilibré leurs portefeuilles par des placements massifs dans les circuits financiers privés dont l'activité atteint ainsi un sommet en $1826^{48}$. Ces conséquences divergentes des courtes périodes d'emprunt massif pendant la première moitié du $\mathrm{XIX}^{\mathrm{e}}$ siècle expliquent qu'on ne retrouve pas alors l'effet d'éviction limité mais net repéré au siècle précédent.

Ajoutons que, si les études parisiennes ont largement tiré parti du regain d'activité provoqué par le Milliard des émigrés, ce fut pour elles un succès éphémère. En tout cas, elles n'ont pas saisi l'occasion de cette redistribution pour réorganiser leurs prestations et pouvoir concurrencer les banques. Les fonds n'ont transité qu'une fois par le notariat parisien. Par la suite, ils ne réapparaissent plus dans les études et il faut donc supposer soit que leur gestion fut confiée à des intermédiaires plus novateurs, soit qu'ils furent placés en province.

Car, outre l'affaiblissement du crédit privé à Paris après la Révolution et l'effacement de ses liens avec le crédit public, l'analyse conduit à souligner une troisième rupture au XIX ${ }^{e}$ siècle : celle qui est intervenue entre la province et Paris. Si partielles que soient nos informations, elles suffisent pour opposer à la stagnation du crédit notarié dans la capitale sa croissance dans le reste du pays. Après avoir lui aussi beaucoup fléchi à la suite de la Révolution, le crédit non parisien s'est développé à nouveau au point de détenir en 1840 une part du marché supérieure à celle qui était la sienne à la fin de l'Ancien Régime. Même compte tenu de l'augmentation de la population sur ce demi-siècle, la progression reste nette (Paris mis à part, le stock de prêt par tête se serait accru d'environ $40 \%$ ) : les effets de la Révolution ont été effacés. Hors de la capitale, les échanges financiers suivaient, voire précédaient le rythme de la croissance économique. En tout cas, Paris n'a pas été, loin s'en faut, le moteur du redémarrage des circuits financiers.

Les performances des différents marchés locaux ont certes été inégales au point d'être parfois pires encore que dans la capitale. Parmi les six marchés examinés, tel fut le cas de celui de l'Isle-sur-la-Sorgue. Mais tous suivirent leur rythme propre, la diversité de leurs évolutions renvoyant aux multiples changements des rapports entre villes et campagnes et à la disparité des développements régionaux. De plus, les particularités de chacun d'eux les ont amené à réagir différemment aux chocs que tous ont subis. Ainsi, l'importance très variable des rentes perpétuelles dans les portefeuilles locaux d'Ancien Régime explique sans doute que certains marchés aient plus souffert que d'autres de l'inflation révolutionnaire. Toutefois le crédit dans les régions de Maubeuge et de Nuits-Saint-Georges progresse au

48. Si l'on limite la comparaison aux volumes nominaux des prêts, l'année 1826 dépasse même les plus hauts niveaux du XVIIr siècle. Naturellement, il n'en va pas de même si l'on compare les stocks. 
même rythme au XIX ${ }^{\mathrm{e}}$ siècle alors qu'avant la Révolution la rente prédominait dans le premier cas et l'obligation dans le second. D'autre part, là où la Révolution a provoqué des migrations des élites locales et modifié des circuits commerciaux, certains marchés ont durablement perdu la place qu'ils occupaient dans la hiérarchie des circuits du crédit.

Partout cependant la taille moyenne des actes a nettement augmenté et la durée des engagements s'est réduite. Comme les Parisiens, les réseaux provinciaux se sont efforcés de s'adapter aux conditions nouvelles et de trouver un équilibre entre coûts de transaction et risque. Eux aussi ont cherché à augmenter la taille des prêts et se sont montrés plus sélectifs dans le choix des emprunteurs. Toutefois, bien que très générale, cette logique qui tendait à évincer les petits prêteurs et plus encore les petits emprunteurs semble avoir plus spécialement affecté le marché parisien.

Le début du XIX ${ }^{\mathrm{e}}$ siècle est ainsi un moment où l'évolution du crédit s'écarte de la tendance à la centralisation qui avait été la sienne jusque-là. Il ne s'ensuit pas que les échanges de capitaux de région à région aient été plus faibles qu'auparavant. Même sur des marchés en crise comme celui de l'Islesur-la-Sorgue ${ }^{49}$ les mouvements de capitaux inter-locaux s'intensifient. C'est aussi le cas de Paris dont le marché s'est ouvert plus largement aux provinciaux (voir tableau 2). Pour autant qu'on en puisse juger par notre échantillon, pendant le XVIII siècle la capitale attirait plus d'argent de la province qu'elle ne lui en prêtait. Après la Révolution, ceci cesse d'être le cas mais la contribution des prêteurs provinciaux au marché privé augmente beaucoup au début du XIX ${ }^{\mathrm{e}}$ siècle, pour les gros contrats en particulier. De très nombreux prêts de plus de 100000 francs concernent alors un prêteur ou un emprunteur non parisien. Une certaine centralisation du crédit à grande échelle se serait donc maintenue alors que de plus petites affaires restaient surtout locales et alimentaient la masse croissante des transactions provinciales.

Depuis le Consulat jusqu'au Second Empire, l'absence ou la faiblesse supposées du crédit à long terme en France et les remèdes qu'il fallait y apporter ont alimenté un interminable débat. La plupart des intervenants ont plaidé pour une participation accrue de l'État dans le marché du crédit privé. Même les plus réservés soulignaient les lacunes et les défauts du système hypothécaire ainsi que la faiblesse des établissements bancaires privés. Les propositions de réforme visaient à concentrer les ressources afin de réduire les risques des épargnants tout en souhaitant favoriser l'accès des petits emprunteurs au crédit. Mais ce second aspect du programme n'eut pas la portée du premier. Comme on l'a vu, autant le crédit notarié se portait mal à Paris, autant sa vitalité était forte dans le reste du pays. Dans ces conditions, assurer une meilleure concentration des capitaux devenait l'objectif prioritaire d'une réforme. Dans le cas du Crédit Foncier dont la création en 1852 fut l'une des principales réponses données à ce long débat, on constate que l'éventail de ses créditeurs a été beaucoup plus ouvert que celui de ses emprunteurs. De même, l'établissement a longtemps attendu avant de se développer en province dont il tirait pourtant une part de ses

49. J.-L. Rosenthal, «The French Revolution and Financial Markets: A Look Beyond Government Debt », article à paraitre, 1994. 
ressources ${ }^{50}$ et si certains de ses prêts sont allés à l'agriculture ou à des activités industrielles décentralisées, l'essentiel s'est dirigé vers l'immobilier parisien. A en juger par cet exemple, l'émergence de banques nationales a donc moins changé le volume du crédit qu'il n'a amené de ressources dans la capitale. Tout porte à croire que les réformes proposées dès le début du XIX ${ }^{\mathrm{e}}$ siècle avaient le même but.

Très intenses au XVIII ${ }^{\mathrm{e}}$ siècle, les échanges financiers qui passaient par les réseaux notariaux parisiens ont beaucoup fléchi après la Révolution. Toutefois, comme les sommes qu'ils prêtaient jusque-là n'ont pu être thésaurisées, il est certain que, dans la capitale, de nouveaux circuits se sont, en partie au moins, substitués aux anciens. Ceux-ci se sont au contraire mieux maintenus dans le reste du pays. Depuis longtemps, ils y avaient organisé un système financier omniprésent et presque partout bien développé si l'on tient compte des différences de revenus. Ils avaient sans doute gardé des structures lourdes et lentes dans la mesure où ils continuaient à pratiquer un crédit personnel où les participants potentiels appartenaient à un réseau particulier. De même, leur activité était restée principalement locale parce que la circulation d'informations fiables sur les emprunteurs ne se faisait qu'à l'intérieur d'un espace restreint. Ainsi, jusqu'à la Révolution, le marché parisien, le plus ouvert de tous, ne plaçait-il guère plus du dixième de ses ressources à l'extérieur.

Pourtant, malgré les risques et les coûts qu'il impliquait, ce système a été assez souple pour permettre une intégration partielle des marchés financiers à l'intérieur de l'espace français et européen. Les poussées de demande de fonds par l'État qu'il a été à même d'absorber en organisant des glissements de capitaux, l'ont moins affaibli qu'elles ne lui ont donné l'occasion de s'étendre. En tout cas, une telle organisation financière a été amplement suffisante pour assurer une certaine mobilité des capitaux. Et la croissance qu'elle a connue (jusqu'à la fin de l'Ancien Régime à Paris et bien plus longtemps ailleurs) atteste pour le moins un certain succès.

Pour partie ce système évoluait au rythme imperceptible de transformations qui conditionnaient les pratiques d'épargne (le vieillissement de la population par exemple). Mais il s'est révélé surtout très sensible aux changements de court terme et particulièrement aux événements politiques qui avaient de fortes retombées sur son fonctionnement et son activité. Le marché parisien devait s'adapter aux émissions publiques, réagir aux décisions financières de l'État, voire les anticiper et, de proche en proche, ses orientations rejaillissaient sur bien d'autres marchés. Mais ce sont de grands chocs politiques comme la Révolution qui ont introduit les innovations les plus profondes. Par leur portée très générale, ils étaient plus que tout autre événement susceptibles de modifier réellement les pratiques dans une activité comme le crédit où les économies de coopération sont importantes. Ainsi, au delà d'une récession aiguë qui se prolongea jusqu'à la Restauration, les

50. J.-P. Allinne, Banquiers et bâtisseurs. Un siècle de Crédit Foncier en France, 1852-1940, Paris, Éd. du CNRS, 1983. 
bouleversements politiques de 1789 à 1798 provoquèrent dans la capitale une crise structurelle du crédit privé qui ne s'était pas estompée au milieu du siècle. L'intérêt d'une étude couvrant une période suffisamment longue est alors de mettre en évidence qu'il s'agit précisément d'une crise structurelle subie par un secteur qui fut longtemps très actif. Contrairement à l'image fixée depuis Gershenckron, le système financier français n'est pas né tardivement des décisions du Second Empire. Jusque-là, parce qu'il était surtout resté organisé dans un cadre jugé a priori archaïque comparé à celui des banques, il est demeuré dans l'ombre au point d'être réputé inexistant ou subalterne. Il avait pourtant amplement prouvé, sous l'Ancien Régime à Paris et après encore en province, sa capacité d'adaptation et de croissance. Mais son histoire heurtée portait encore au milieu du siècle la marque de l'événement révolutionnaire.

\author{
Philip HofFMaN \\ California Institute of Technology \\ Gilles Postel-Vinay \\ INRA \\ Jean-Laurent RosENTHAL \\ University of California - Los Angeles
}




\section{MODERNISATION DES SOCIÉTÉs TRADITIONNELLES}

\section{Annexe}

Tableau A - Taille et durée des prêts par période

Taille des prêts (Livres/Francs)

\begin{tabular}{|c|c|c|c|c|c|c|}
\hline & \multirow[b]{3}{*}{ Perpet. } & \multicolumn{4}{|c|}{ Taille des prêts (Livres/Francs) } & \multirow{2}{*}{$\begin{array}{l}\text { Durée des } \\
\text { Obligations }\end{array}$} \\
\hline & & \multicolumn{2}{|c|}{ Emprunts privés } & \multicolumn{2}{|c|}{ Emprunts publics } & \\
\hline & & Oblig. & Viag. & Perpet. & Viag. & $(e n$ \\
\hline & - & - & - & - & - & - \\
\hline 1740 & 8500 & 5400 & 7000 & 20000 & 6600 & 2,8 \\
\hline $1755-1765$ & 10100 & 7500 & 8600 & 13700 & 1300 & 3,4 \\
\hline $1766-1775$ & 10800 & 8500 & 5500 & 12300 & 2300 & 3,6 \\
\hline $1776-1789$ & 13300 & 18400 & 9000 & 10700 & 3900 & 4,2 \\
\hline $1798-1806$ & 13400 & 9100 & 9000 & - & - & 2,1 \\
\hline $1807-1815$ & 8000 & 10900 & 9600 & - & - & 2,9 \\
\hline $1816-1820$ & 6700 & 15900 & 9300 & - & - & 3,3 \\
\hline $1821-1830$ & 7000 & 21700 & 10900 & - & - & 3,4 \\
\hline $1831-1841$ & 7000 & 18000 & 8800 & - & - & 4,9 \\
\hline
\end{tabular}

Sources : Echantillon d'actes des études IX, XXI, XLIII, LXII, LXX, LXXVIII et CXV.

Tableau B - Résultats des sondages en coupes (Sommes prêtées par étude, moyennes en milliers de livres/Francs)

$\begin{array}{cccc}\text { Moyennes } & \text { Ecart-Type } & \text { Mini } & \text { Maxi } \\ \text { (9 études })(21 \text { études }) & \text { (30 études) }\end{array}$

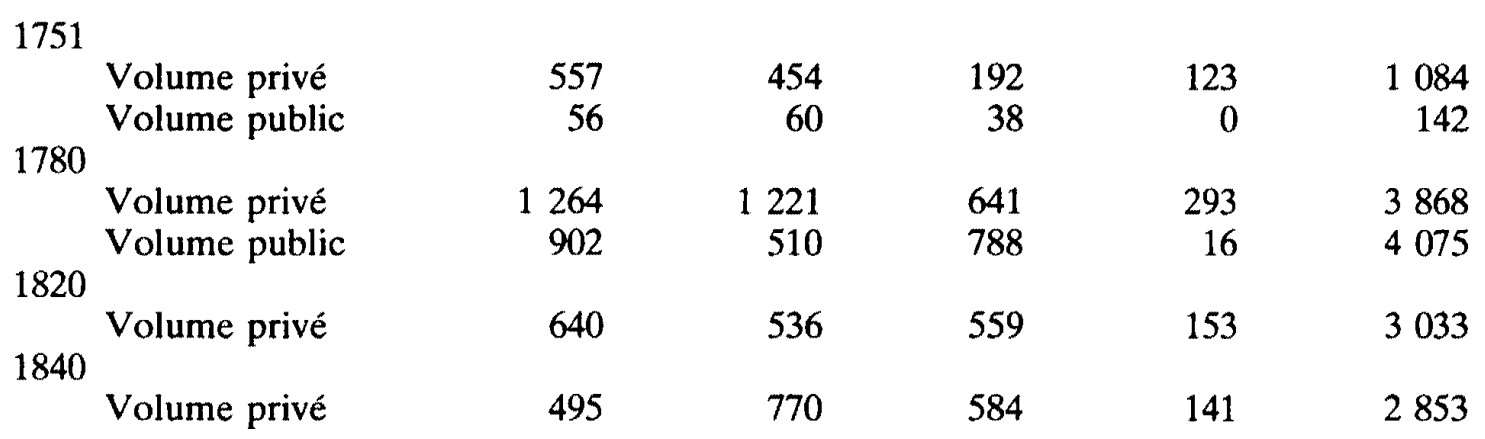

Sources : voir texte 